\title{
Heuristic Rule-Based Genetic Algorithm for Large-Size High-Constrained Single-Stage Multi-Product Scheduling with Parallel Units
}

\author{
Yaohua He, Chi-Wai Hui* \\ Chemical Engineering Department, Hong Kong University of Science and Technology, \\ Clear Water Bay, Hong Kong, P. R. China \\ (E-mail address: keyhhe@ust.hk, kehui@ust.hk Tel: +852-23587137)
}

\begin{abstract}
This paper presents a heuristic rule-based genetic algorithm (GA) for large-size high-constrained single-stage multi-product scheduling problem (SMSP) with parallel units. SMSP can be classified into two types: type one is the problem with changeover and process constrains (CP constraints); type two is that without CP constraints. This paper is mainly concerned with type one. SMSP has been widely studied by the researchers. Most of them used mixed-integer linear programming (MILP) formulation to solve the problem. When the problem size increases linearly, the computation time of MILP will increase exponentially. It is very difficulty for MILP to solve large-size problem. Therefore, the researchers often use small instances to illustrate their MILP model. To solve large-size classical scheduling problems, meta-heuristic methods, like GA, are widely used. However, due to the CP constraints in SMSP, it is not easy to obtain feasible solutions in heuristic search. Hence, some authors concluded that GA is not suitable for this type problem, especially large-size problem. Indeed, if quite a few CP constraints exist, it will take GA plenty of time to generate feasible solutions. Nevertheless, by replacing the forbidden changeovers and processes with a large penalty numerical value, the problem is changed to be type two. By this penalty method, the search time of GA to large-size SMSP under CP constraints is reduced greatly. In our proposed method, GA is combined with heuristic rule that greatly reduces the solution space and the search time. Through comparison of computation results of MILP, random search and GA, GA demonstrates promising performance in solving large-size high-constrained SMSP.
\end{abstract}

Keywords: Parallel Unit Scheduling; Mixed-Integer Linear Programming; Random Search; Heuristic Rule; Genetic Algorithm

\section{Introduction}

Short-term scheduling plays an important role in the planning of batch operations to increase the efficiency batch plant. There is a large body of literature on process scheduling.

Reklaitis and Mockus (1995) proposed a non-uniform time discretization model, in which binary variables were applied to represent the start and stop events for the various recipe tasks. They used the randomized heuristic approach (Mockus and Reklaitis, 1996) to reduce the number of binary variables.

Pinto and Grossmann (1995) presented a continuous time MILP model for short-term scheduling of multistage batch production with parallel production lines. They used the concept of parallel time coordinates for units and tasks. Furthermore, Pinto and Grossmann (1996) proposed an alternate model in which the pre-ordering of orders was imposed explicitly, by applying an alternative representation of the time slots for the units. This resulted in a significant reduction in

*To whom correspondence should be addressed. E-mail: kehui@ust.hk 
computational time consumed.

Cerda et al. (1997) proposed a continuous time MILP model for short-term scheduling of a single stage multi product batch plants with parallel units. They used tri-index decision variables as well as the concept of predecessor and successor to describe the order assignment to various production units while taking into account sequence dependent changeover constraints. To deal with large size problems, they proposed heuristics, such as the order preordering, to reduce the number of feasible predecessors for each order.

On the basis of the notation of time slot, Karimi and Mcdonald (1997) proposed two models for parallel semicontinuous processes considering the sequence-dependent setup times, orders, and their corresponding due dates in order to minimize the inventory. The major advantage of the formulation is that it can incorporate fixed time events such as due dates while using continuous representation of time.

Ierapetritou et al. (1998) presented an effective continuous time formulation for short-term scheduling of multi-purpose batch plants. The novel feature of this formulation was the de-coupling of task events from unit events, which greatly reduced the number of decision variables. The major limitation of this formulation was that it required pre-ordering of all the orders in advance, which restricted its application to specific problems. Furthermore, the model has difficulty in accounting for order sequence dependent constraints.

Hui et al. (2001) presented a general formulation for SMSP. The proposed formulation applies three sets of bi-index variables to handle order sequence dependent constraints either with or without imposing pre-ordering heuristics. The main advantage of this formulation compared to the other recently proposed formulations is the significant reduction in the number of binary variables and consequently shortening the solution time. The authors claimed their method suitable for handling large-size industrial problems.

Chen et al. (2002) develops a short-term scheduling model for SMSP using the continuous-time domain representation and the notation of time slot. When the model is developed, the allocation of orders and units to time slots is represented by two sets of binary variables. The MILP model not only involves fewer binary variables than any other model based on the notation of time slot (Pinto and Grossman, 1998) but also can be used to optimize several types of objective functions.

Wu and Ierapetritou (2004) presented a new framework for the solution of scheduling problem based on a continuous-time formulation and the idea of operating periodicity. It determined the optimal cyclic schedule as well as the optimal cycle length for multipurpose batch plant. Research in this paper extended the work by Ierapetritou and Floudas (1998a) and Ierapetritou and Floudas (1998b) based on the STN representation, and develops the cyclic scheduling formulation with the inherited advantage of using few binary variables.

Cafaro and Cerda (2004) presented A new MILP continuous-time approach for the scheduling of a single multi-product pipeline transporting refined petroleum products from a single oil refinery to several distribution centers. By adopting a continuous representation in both time and volume, a more rigorous problem description and a severe reduction in binary variables, constraints and CPU time have simultaneously been achieved. The use of a rigorous problem representation brings about several advantages.

Ghaeli, Bahri, Lee et al. (2005) presented a novel approach based on the timed place petri-net (TPPN) formulation for the scheduling of batch plants. It was shown that the changes in the 
markings of a TPPN model completely describe the evolution of different operations. The optimal schedule could be obtained by searching through the reachability tree of the TPPN model using branch and bound method. The heuristic algorithm is applied in order to decrease the number of searches through the reachability tree.

Heuristic rule is a widely used technique cutting down the size of an MILP model. Pinto and Grossmann (1996) greatly cut down the size of the model (Pinto and Grossmann, 1995) for multistage batch plants through imposing the preordering constraints (heuristic rules) into it. The formulation of the simplified model involves only decisions of assignment of orders to units and timing of each processing task. However, the preordering constraints affect the optimality of the scheduling model. Cerda et al. (1997) reduced the size of the scheduling model for single-stage multiproduct batch plants with parallel lines by introducing some heuristic rules, so that the feasible predecessors of an order are pruned. These heuristic rules are also used by Mendez et al. (2000a and 2000b) and Hui and Gupta (2000). However, if these heuristic rules are used, the optimal solution cannot be obtained, especially when makespan is minimized. The MILP model proposed by Chen et al. (2002) introduced some heuristic rules that can cut down the size of the model and has no effect on the optimality of the scheduling problem.

All of the above methods are MILP actually, focusing on small-size problems. When the problem size increases linearly, the computation time of MILP will increase exponentially. It is very difficulty for MILP to solve large-size problem. Although some researchers (Pinto,Turkay et al., 1998; Hui et al., 2001) claimed their MILP models suitable for large-size problems, but the solution to large-size problem is far from the optimum, even if the algorithm ran for very long time, say several hours.

Meta-heuristic methods, although they cannot guarantee a global optimum, can provide near-optimal solutions within moderate or acceptable computing time. Therefore, meta-heuristic methods are more suitable for large-size problems. There has been a large body of literature on meta-heuristic methods for classical scheduling problems; but only several papers on genetic algorithms (GA) (Chen et al., 1996; Kim et al. 1996; Jou, 2005), simulated annealing (SA) (Das et al., 1990; Ku and Karimi, 1991), list-based threshold-accepting algorithm (LBTA) (Lee et al., 2002) for process scheduling problems. Some authors believe that, due to the high complexity and constraints of process scheduling, meta-heuristic methods, such as genetic algorithm and tabu search, are not suitable for process scheduling problems. Their reason is that the original feasible solution(s) for evolution is or are not easy to be achieved.

In this paper, we present genetic algorithm based on heuristic rule for large-size high-constrained SMSP. During recent years, Hui et al. (2001) and Chen et al. (2002) studied this problem, illustrating their MILP models by small-size instances. In our work, first, the size of the instances was enlarged, and from the computation results of MILP method provided by Hui et al. (2001), we could see the difficulties of MILP for large-size problem. Then random search (RS) based on heuristic scheduling rule was employed for the same instances. Subsequently, GA based on heuristic scheduling rule was used for the same instances as before. From the computation results by MILP, RS and GA, it can be seen that GA performs much better than MILP and RS. These results encourage us to carry out further research on rule selection and search technique improvement. 


\section{Problem Definition}

A fixed number of production units (forming a set of units: $U$ ) are available to process all customer orders (forming a set of orders: $O$ ). Assume the number of production units is $M$, and the number of customer orders is $N$. Each order involves a single product, requiring a single processing stage, has a predetermined due date, and can only be processed in a subset of the units available. The production capacity of a unit depends on the order processed. The size of an order may be larger than the size of a batch, requiring several batches to satisfy an order. Batches of the same order are processed consecutively in the same unit. A production unit processes only one batch at a time. The batch-time of an order is fixed and production unit dependent. During the transition of production orders, time is required for the preparation of the unit for the changeover. The changeover time is sequence-dependent. There are forbidden changeovers and forbidden processes in the problem, named as CP constraints. The objective of the scheduling is to minimize the total tardiness or schedule makespan by the assignment of orders to units while satisfying all the above constraints.

$N$ orders: Each order is a product requiring a single processing stage;

Each order has a predetermined due date $\left(d_{j}\right)$;

Can only be processed in a subset of the units available (process time $p_{j u}$ );

Not all the orders can be processed by any unit (let process time $p_{j u}=0$ );

Each order maybe has a release time (order release time $o r_{i}$ ).

$M$ units: A production unit processes only one order at a time;

When changing from one order to another order, time is required

(changeover time $c_{i j}$ );

Not all the orders can be changed over to any order (let changeover tome $c_{i j}=\mathbf{0}$ );

Each unit maybe has a release time (unit release time $u r_{u}$ ).

Objective: to minimize makespan $C_{\max }$ or total tardiness $T$.

Example 1: 50 orders over 4 units ( $N=50, M=4$, with CP constraints);

Changeover time matrix: $c_{i j}$, some of $c_{i j}=0$;

Due date: $d_{j}$;

Order release time: ${ } r_{i}$;

Unit release time: $u r_{u}$;

Process time matrix: $p_{j u}$, some of $p_{j u}=0$.

Example 2: 100 orders over 8 units ( $N=100, M=8$, without CP constraints);

$c_{i j}$ and $p_{j u}$ are generated randomly;

Changeover time matrix: $c_{i j}$, no $c_{i j}=0, c_{i j} \in(0.10,2.00)$;

Due date: $d_{j}$;

Order release time: or $_{j}$;

Unit release time: $u r_{u}$;

Process time matrix: $p_{i u}$, no $p_{j u}=0, p_{j u} \in(5.0,20.00)$.

The data of Example 1 are recorded as Table $1 \sim 4$ in Appendix. Order process time $p_{j u}$ is calculated from the order size $Q_{j}$, batch size $B_{j u}$ and batch time $b_{j u}$ : 


$$
p_{j u}=\text { ceil }\left(\frac{Q_{j}}{B_{j u}}\right) \times b_{j u}
$$

\section{Difficulties for MILP}

\subsection{Computation results by MILP}

To solve SMSP, the procedure of MILP given by Hui et al. (2001) is as follows: establish the MILP model first, including objective function and constraints, and then solve the model by GAMS, a commercial solver. The model looks very complicated. The scheduling objective is to minimize makespan or total tardiness. For makespan, the objective function is:

$$
\min C_{\max }=\max \left\{C_{1}, \quad C_{2}, \quad \ldots, \quad C_{N}\right\}
$$

where $C_{j}$ is the completion time of order $j$;

for total tardiness, the objective function is:

$$
\min \quad T=\sum_{j=1}^{N} T_{j}
$$

where $T_{j}$ is the tardiness of order $j: T_{j}=\max \left\{C_{j}-d_{j}, 0\right\}$.

Table 5 is the computation results of Example 1 by MILP. In case a, all the unit release times and the order release times are null. In case b, the unit release times and the order release times are finite, that is to say, some of the release times are zero, and some of them are with finite value (see Table 1 and Table 3). The computation tests were run on computer with Intel (R) Pentium (R) M $1500 \mathrm{MHz}$ CPU and 256M memory.

\subsection{Difficulties for MILP}

Through analyzing the computation results in Table 5, it could be seen that:

(1) For small-size instances, MILP can get the optimal solution of the problem in short time, for example, for problem with 10 orders over 4 units, computational procedure will stop with a small number of iterations when optimal solutions has been obtained.

(2) For large-size instances, MILP cannot get the optimal solution of the problem in acceptable time. For 50-order problem, iterations increased from 100000 to 1000000, however, the total tardiness changed very little, and the makespan had no change. This verifies that: when the problem size increases linearly, the computation time of MILP will increase exponentially.

(3) If the problem highly constrained, the solution for it will be obtained more rapidly by MILP. The reason for this is that the CP constraints cut some solution space for MILP. The more the constraints, the more rapidly MILP method finds out the solution.

From the following three Gantt charts (see Fig. 1 3), we can see that: an optimal schedule corresponds to an uniform Gantt chart with small time intervals; on the contrary, a non-optimal schedule corresponds to a Gantt chart with big time intervals.

All in one, MILP seems not suitable to solve large-size high-constrained SMSP. 


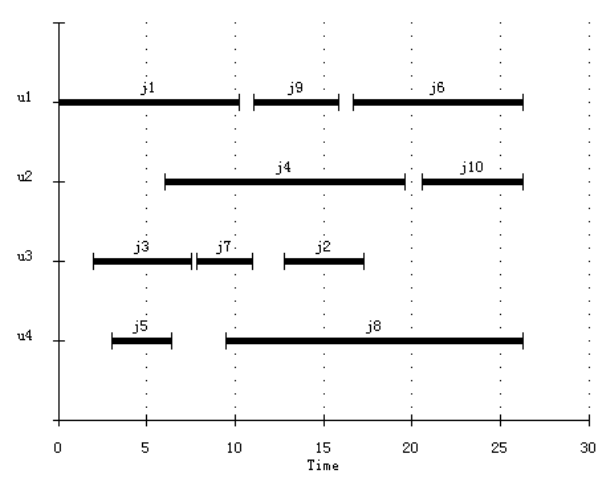

Fig. 1 Gantt chart of an optimal schedule for example $1 \mathrm{~b}$ (10 orders over 4 units) by MILP (makespan=26.25)

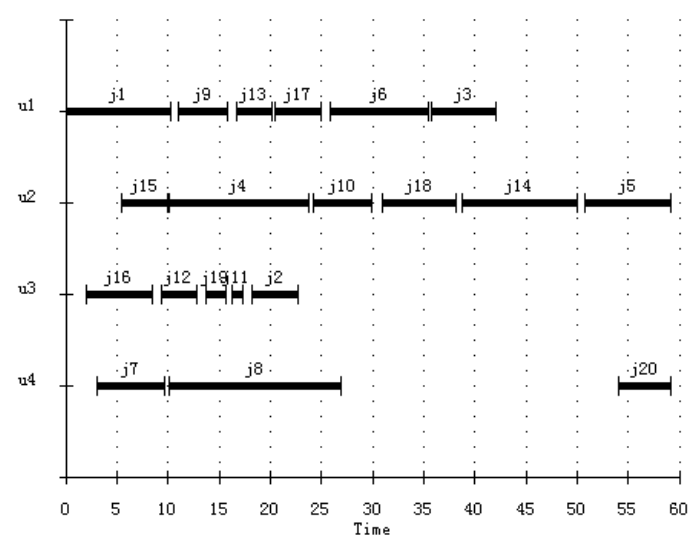

Fig. 2 Gantt chart of a schedule for example 1b (20 orders over 4 units) by MILP (makespan=59.24)

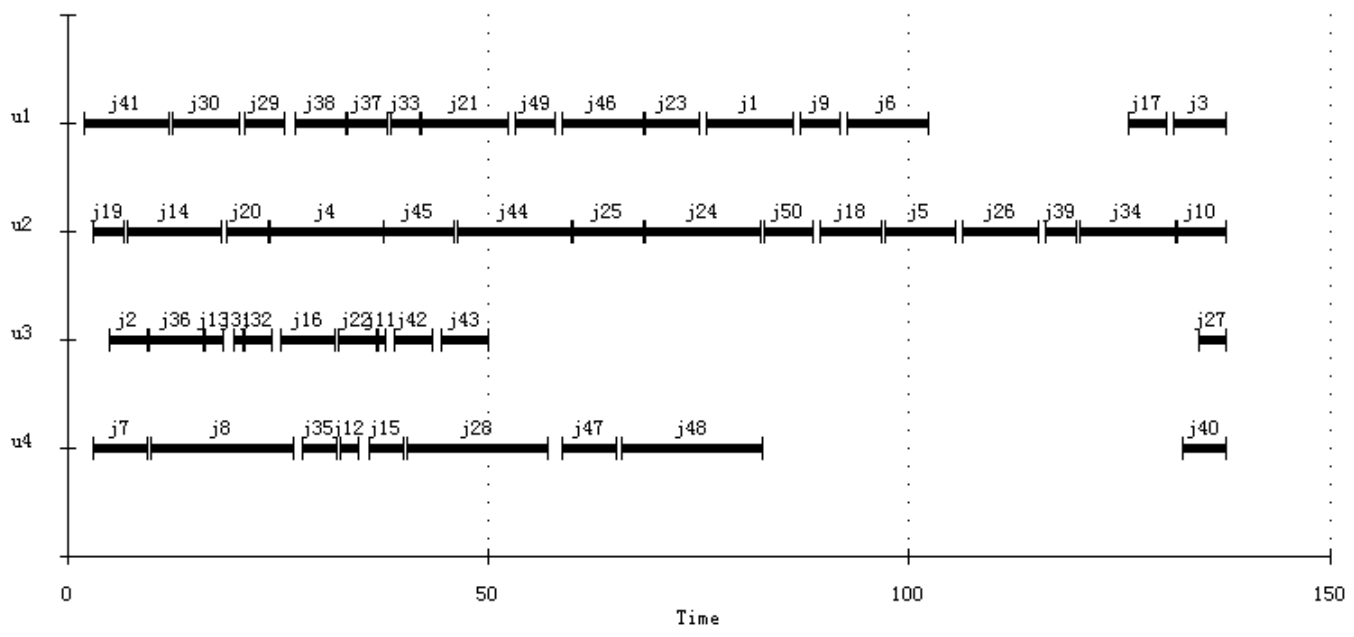

Fig. 3 Gantt chart of a schedule for example 1b (50 orders over 4 units) by MILP (Makespan=137.65) 


\section{Random Search Based on Heuristic Rule}

Due to difficulties of MILP for large-size high-constrained SMSP, the preferred approach in industry is the use of scheduling rules, such as shortest processing time first (SPT) rule, earliest due date first (EDD) rule. According to a scheduling rule, the customer orders are sequenced in decreasing priority order, and then one by one assigned to the production units according to unit selection rule. In SMSP without CP constraints, one feasible solution can be acquired by using scheduling rules. However, in SMSP with CP constraints, due to the CP constraints, feasible solution may not be acquired by using scheduling rules.

In order to get feasible or much better solutions, random search (RS) could be used: the customer orders are randomly sequenced, and then one by one assigned to the production units according to the same unit selection rule. Through RS, a number of solutions can be obtained, among which feasible or much better solutions may be found.

\subsection{Representation of a Random Solution}

Serial numbers $1,2,3, \ldots, N$ are used to denote $N$ orders that will be assigned over $M$ units. A random order string $\pi=\left(\pi_{1}, \pi_{2}, \pi_{3}, \ldots, \pi_{N)}\right.$ is produced, $\pi_{\mathrm{i}} \in\{1,2,3, \ldots, N\}, i=1,2,3, \ldots, N$. And then, from $\pi_{l}$ to $\pi_{N}$, one by one, each order will be assigned over the units according to a certain heuristic rule (unit selection rule). As a result, considering the constraints given, if all of the orders are able to be assigned to the units, then $\pi$ stands for a feasible solution, and the objective value is $f(\pi)$; otherwise, $\pi$ is infeasible. (In fact, the order string is called chromosome in genetic algorithm). As the scheduling objective is makespan or total tardiness, so for makespan:

$$
f(\pi)=C_{\max }=\max \left\{C_{1}, \quad C_{2}, \quad \ldots, \quad C_{N}\right\}
$$

where $C_{j}$ is the completion time of order $j$;

for total tardiness:

$$
f(\pi)=T=\sum_{j=1}^{N} T_{j}
$$

where $T_{j}$ is the tardiness of order $j: T_{j}=\max \left\{C_{j}-d_{j}, 0\right\}$.

\subsection{Heuristic Rules}

To minimize the makespan or total tardiness, the following heuristic rules are available to assign the order (These rules form a candidate rule base that can be enlarged still):

Rule 1:Assign the order on the unit that makes the order's completion time to be as early as possible (earliest completion time, ECT);

Rue 2: Assign the order on the unit that makes the order's start time to be as early as possible (earliest start time, EST);

Rue 3: Assign the order on the unit that make the order's process time on the unit to be the shortest (shortest process time, SPT);

Rue 4: Assign the order on the unit that makes the order's changeover time on the unit is 
the shortest (shortest changeover time, SCT);

Rue 5: Assign the order on the units by random (Random Rule);

Rue 6: Assign the order on the unit that makes the sum of the order's changeover time and process time on the unit to be the shortest (shortest process + changeover time, SPCT).

Fig. 4 is the illustration of how Rule 1 and Rule 2 work. Other rules work similarly.

In Fig. 4, assume that a random order string $\pi=(8,2,5,4,3,7,6,1, \underline{9}, \ldots$, ), and the former eight orders have been assigned over four units, order 9 is to be assigned, as Fig. 4(a) shown.

Before order 9 assigned, the current completion time of Unit 3 is the shortest among those of the four units. According to Rule 2, order 9 should assign over Unit 3 as Fig. 4(d) showed.

If order 9 assigned to each unit, each unit will get a new current completion time, as Fig. 4(b) shown. Comparing the four new current completion times, Unit 2 has the earliest one. According to Rule 1, order 9 should be assigned to Unit2, as Fig. 4(c) shown.

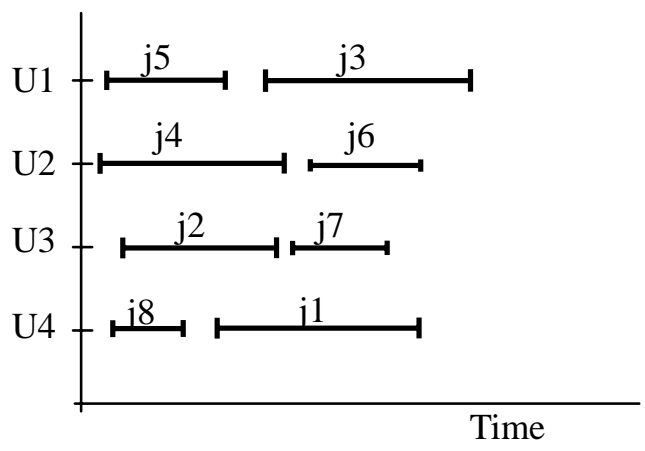

(a) Before order 9 assigned

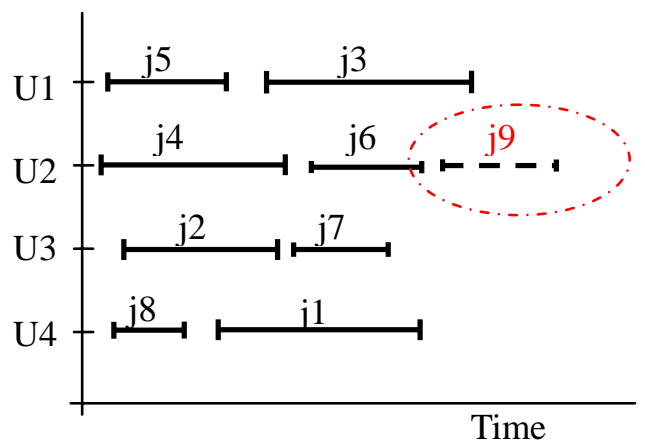

(c) Assigned as Rule 1 required

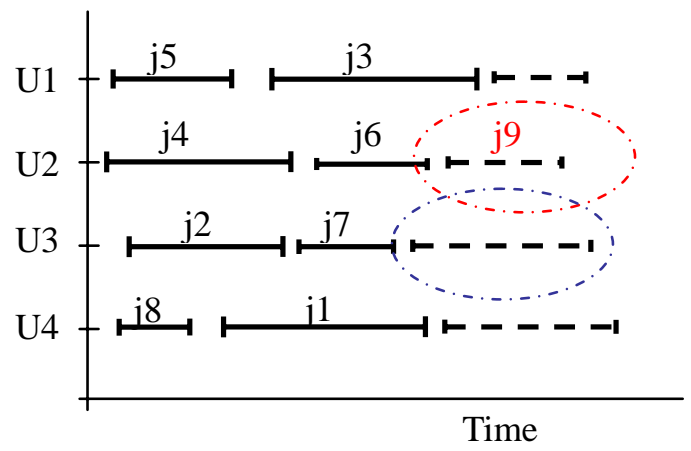

(b) Order 9 to be assigned

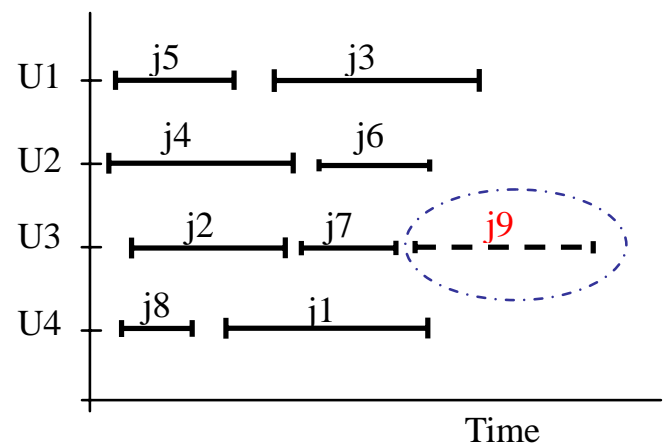

(d) Assigned as Rule 2 required

Fig. 4 Contrast of Rule 1 and Rule 2

According to Rule 1, the orders will be assigned to the units uniformly, so we may judge subjectively that Rule 1 is the best among the above rules. Through simulation experiments, it is also found that Rule 1 is the best among the above rules to SMSP with CP constraints.

\subsection{Schedule Synthesis by Heuristic Rule}

The procedure of schedule synthesis by heuristic rule could be illustrated as Fig. 5. The heuristic rule is the criterion to assign every order in the order string to a unit. If the assignment of any order failed, the order string is unfeasible, then a new order string will be re-generated, until a feasible order string generated. 


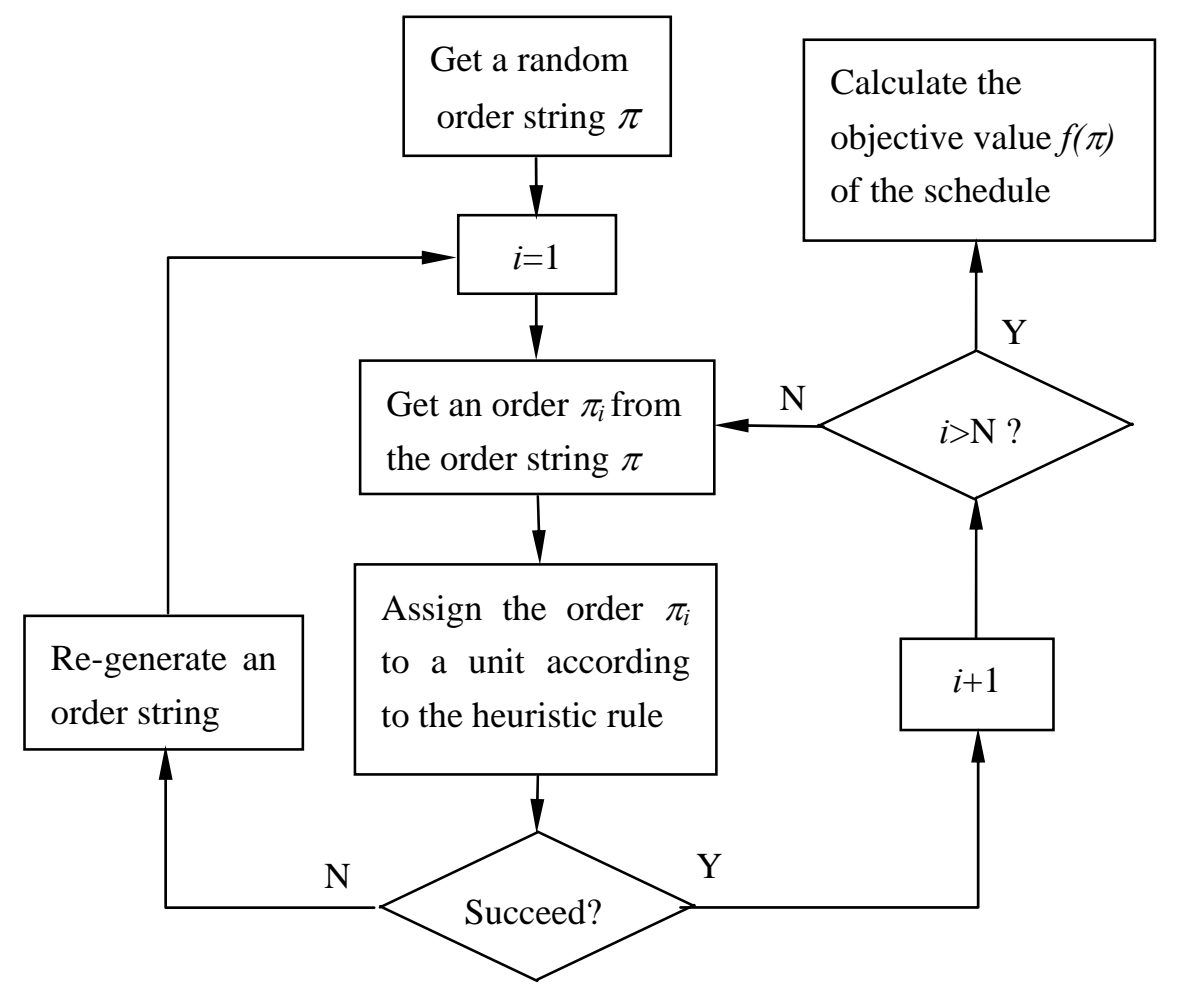

Fig. 5 Flow chart of schedule synthesis by heuristic rule

\subsection{Procedure of the Random Search}

The procedure of the random search, shown as Fig. 6, can be easily understood. The number of random feasible solutions is popsize that is set according to the problem size, see Table 6.

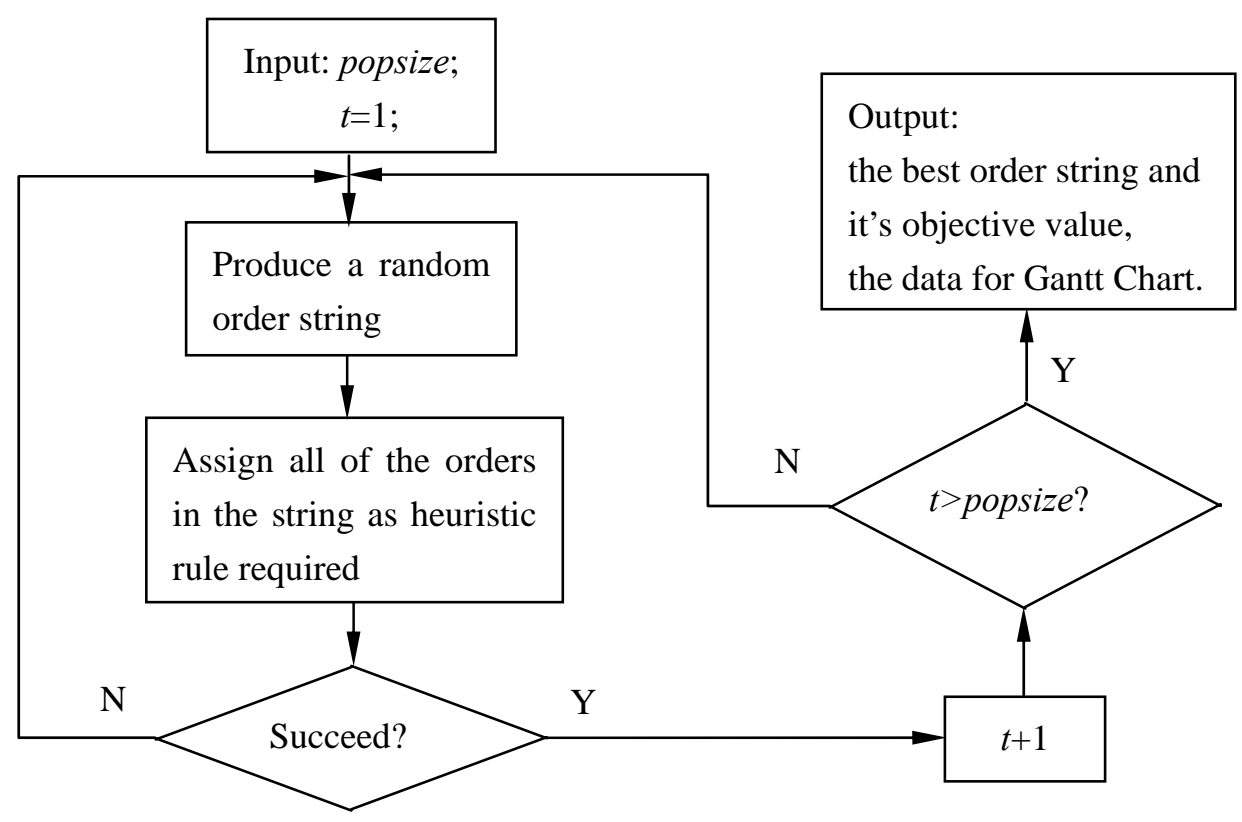

Fig. 3-6 Flow chart of random search 


\subsection{Computation Results by Random Search}

Table 6 is the computation results of Example 1 by random search based on heuristic rule.

From Table 6, it could be found that:

(1) For small-size instances, say that with 10 orders over 4 units, random search can find out the optimal solution like MILP from several hundreds of random feasible solutions.

(2) For large-size instances, random search will spend much time to get a feasible solution, so it takes long CPU time (if the problem without CP constraints, every random string produced makes a feasible solution, so random search can rapidly find out a better solution from the random solutions. This is different from MILP).

(3) Random search obtains better solutions in relatively shorter time than MILP, with the problem size increasing.

(4) A carefully chosen heuristic rule plays a very important role in random search.

Because the heuristic rule (unit selection rule) greatly reduces the solution space, RS performs better than MILP.

\section{Genetic Algorithm Based on Heuristic Rule}

To evolve the random solutions, a heuristic rule-based GA is proposed in this paper. The proposed method combines GA with the heuristic rule (unit selection rule) for specific scheduling objective. At the beginning of GA, a number of random solutions are generated. Through evolutionary mechanism of GA, near-optimal solution can be achieved.

Some authors concluded that GA is not suitable for highly constrained problem, especially large-size highly constrained problem. Indeed, if quite a few CP constraints exist in SMSP, it will take GA plenty of time to generate feasible solutions. However, by replacing the forbidden changeovers and processes with a large penalty numerical value, the problem is changed to be type two. By this penalty method, the search time of GA to large-size SMSP under CP constraints is reduced greatly.

\subsection{Components of Genetic Algorithm}

\subsubsection{Representation of solutions}

In solving scheduling problems by GA, first task is to represent a solution of a problem as chromosome. Permutation-based representation is adopted in this paper. As stated in section 4.1, serial numbers $1,2,3, \ldots, N$ are used to denote $N$ orders that will be assigned over $M$ units. A random order string $\pi=\left(\pi_{1}, \pi_{2}, \pi_{3}, \ldots, \pi_{N}\right)$ is produced, $\pi_{i} \in\{1,2,3, \ldots, N\} . \pi$ is called a chromosome in GA. Fig. 7 is a sample chromosome of 10 -order problem.

$$
\begin{array}{llllllllll}
6 & 2 & 5 & 4 & 8 & 3 & 7 & 1 & 9 & 10
\end{array}
$$

Fig. 7 A sample permutation-based chromosome 


\subsubsection{Generation}

At the beginning of GA, an initial population of chromosomes are randomly generated, named as initial generation. Assume the number of chromosomes in the initial generation is popsize. In each iteration of GA, a new generation will be produced by crossover, mutation and selection.

\subsubsection{Selection}

Selection is the process to select most of the better chromosomes in each generation to crossover, and the rest or some of the rest to mutate. Although the roulette-wheel selection (Goldberg, 1989) is one commonly used technique, but tournament method (Goldberg and Deb, 1991) is adopted in our GA, because in tournament method objective value can be used as selection criterion, not always fitness as roulette-wheel selection. Assume the number of the chromosomes that are selected to crossover is xsize; the number of the chromosomes that are selected to mutate is msize. We may let:

$$
x \text { size }+ \text { msize }=\text { popsize }
$$

The ratio $C_{r}=x$ size/popsize is called crossover rate, often $C_{r} \in[0.5,0.9]$; and the ratio $M_{r}=$ xsize/ popsize called mutation rate, often $M_{r} \in[0.1,0.3]$. So $C_{r}+M_{r}=1$. $C_{r}$ and $M_{r}$ are two important parameters that influence the convergent performance of GA. In general, if $M_{r}$ increases, GA will be convergent to the final solution slowly, so that GA has more chances to find out better solutions. But if $M_{r}$ is too large, GA tends to be like random search.

\subsubsection{Crossover}

There are several methods to crossover (Poon and Carter, 1995). Partial-mapped crossover (PMX) is adopted in the proposed GA, as shown in Fig. 8.

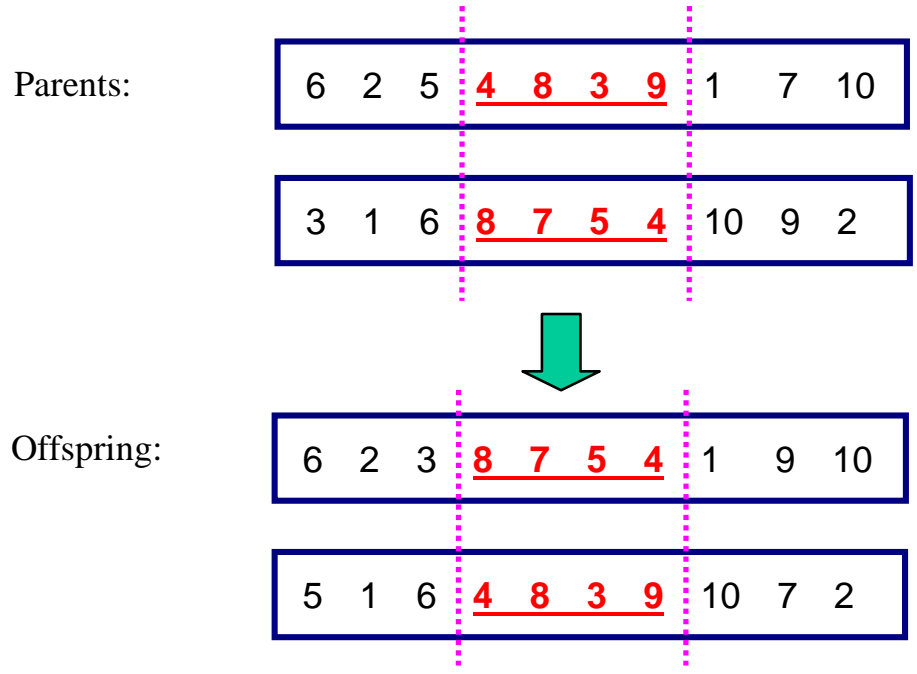

Fig. 8 Illustration of the process of PMX 


\subsubsection{Mutation}

Mutation is the operation to make some genes of the chromosome changed. There are also a number of methods to mutate, such as insertion, swap and reversion, see Fig. 9. Reversion is employed in GA.

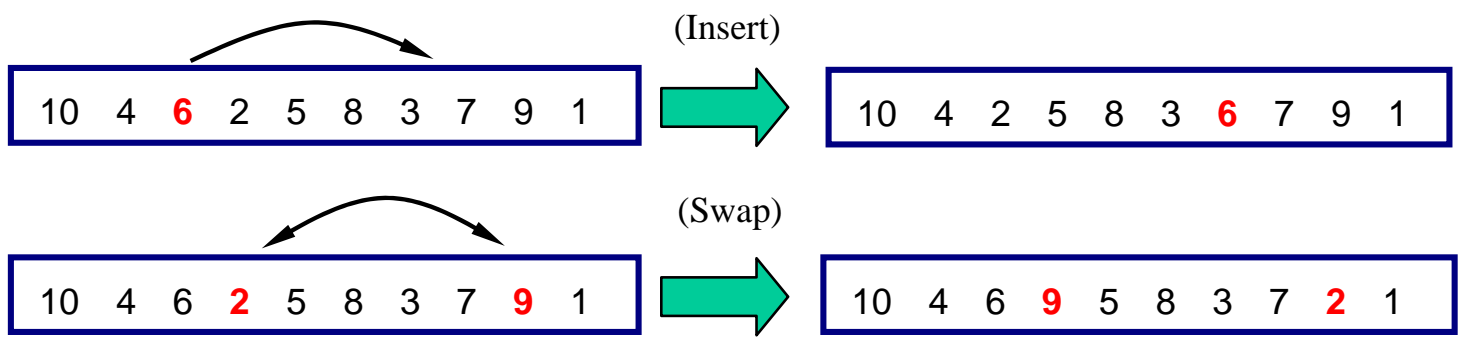

(Reverse the chosen part)

$$
\begin{array}{llllllllll}
10 & 4 & 6 & 2 & 5 & 8 & 3 & 7 & 9 & 1
\end{array}
$$

$\begin{array}{llllllllll}10 & 4 & 6 & 2 & 7 & 3 & 8 & 5 & 9 & 1\end{array}$

Fig. 9 Illustration of the process of mutation

\subsubsection{Termination condition}

The algorithm terminates when no further improvement on the solutions exists (named until-no-improvement). With the algorithm running on, the difference among the chromosomes will tend to be null. That means no further improvement on the solutions exists, the algorithm should terminate.

\subsection{Procedure of Genetic Algorithm}

The whole GA procedure is shown as the Fig. 10. Because of the CP constraints in Example 1, un-feasible chromosomes will surely be generated in the three steps: "Initial generation", "Crossover", "Mutation”. In this case, a new feasible chromosome should be re-generated. So the "Initial generation" step is equivalent to the random search in section 4.

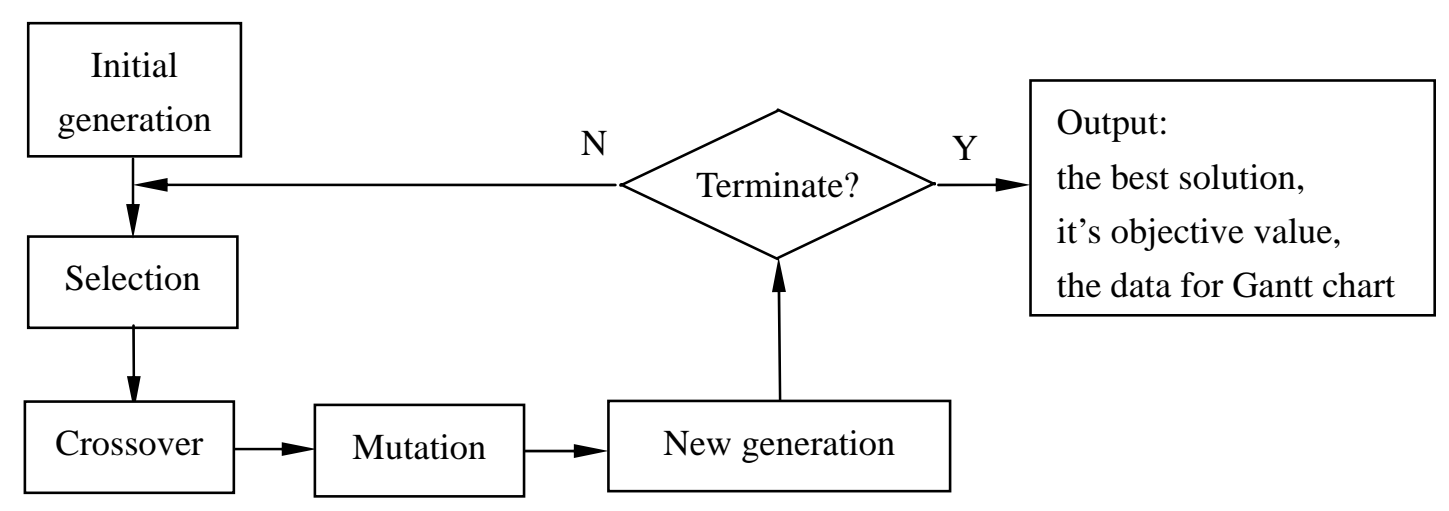

Fig. 10 Flow chart of genetic algorithm 


\subsection{Penalty to CP Constraints}

In solving SMSP with CP constraints, $t$ is found that GA took very long time to get the final solution to large-size problem, say 50 -order problem, see Table 7. The reason is that GA spent relatively long time to obtain feasible chromosomes (or solutions).

In order to increase computing speed of GA, we have taken the following measures: give the forbidden changeover (from one order to another order) or forbidden process (meaning that a order is forbidden to process over a unit) a large penalty numerical value: a great changeover time or process time. For instance, change the null value in changeover time matrix and process time matrix into 100.0. Therefore, every random chromosome of the problem became a legal or feasible one. As a result, the CPU time reduced greatly, furthermore, GA obtained similar solutions as before (see Table 8).

Fig. 11(a) and (b) show the difference between methods with and without penalty to CP constraints. When the forbidden changeovers and processes are substituted by a large penalty numerical value, the problem is changed to be without CP constraints. Hence at the beginning of the algorithm, the objective values of the best chromosome (solution) are much high, but decreasing very steeply. The CPU time of the algorithm is reduced greatly.

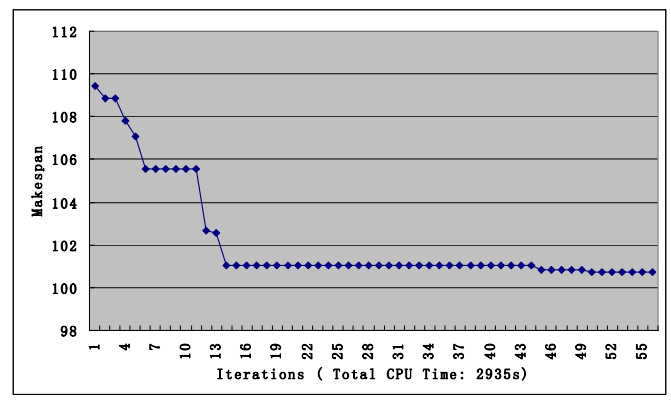

(a) GA process (Example 1b: 50 orders over 4 units)

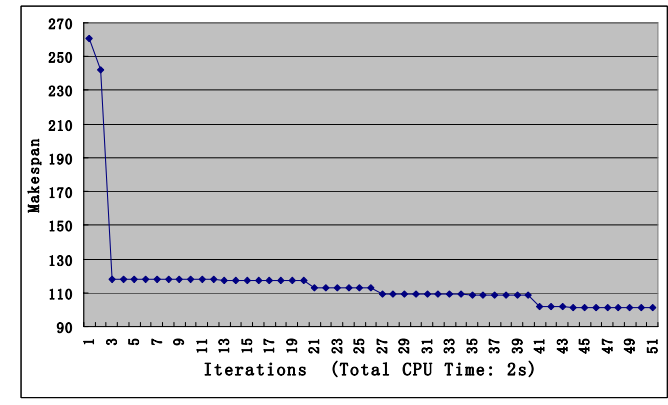

(b) GA process ( Example 1b: 50 orders over 4 units with penalty)

Fig. 11 Contrast of GA with and without penalty to CP constraints

\subsection{Computation Results by Genetic Algorithm}

Table 7 and Table 8 are the results of Example 1 by GA. It could be seen that, for the small-size instances, GA got the optimal solution, as RS and MILP did; with the problem size increasing, GA shows it advantage over RS and MILP. For the large-size instances, GA found much better solutions than RS and MILP.

\section{Comparison the Results of GA, RS and MILP}

Comparing the results, we can easily find that: with the problem size increasing, the distinctions between the performances of GA, RS and MILP become more and more magnificent. For the 50-order problem, the tardiness value achieved by GA is much smaller than that by RS and MILP; the makespan achieved by GA is also much better than that by RS and MILP. As mentioned before, the final schedule obtained by MILP has evident and big time intervals; however, the final schedules obtained by GA and RS are even, uniform and acceptable (see the Gantt charts Fig. 12 and Fig. 13). 


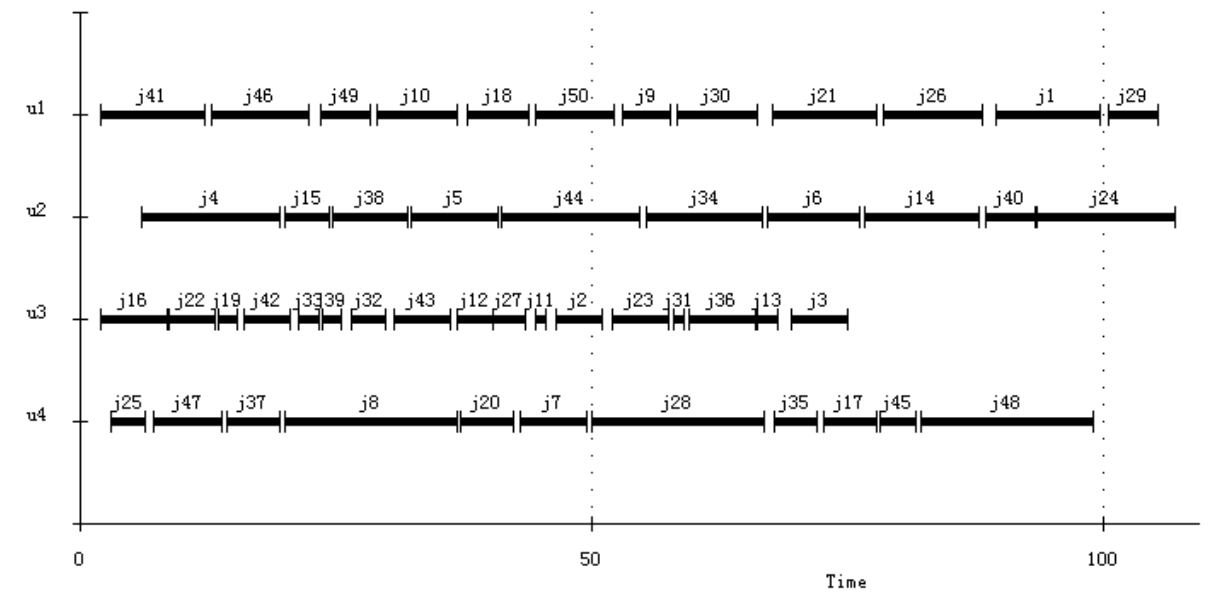

Fig. 12 Gantt chart of a schedule for Example 1b (50 orders over 4 units) by random search (makespan=107.14 )

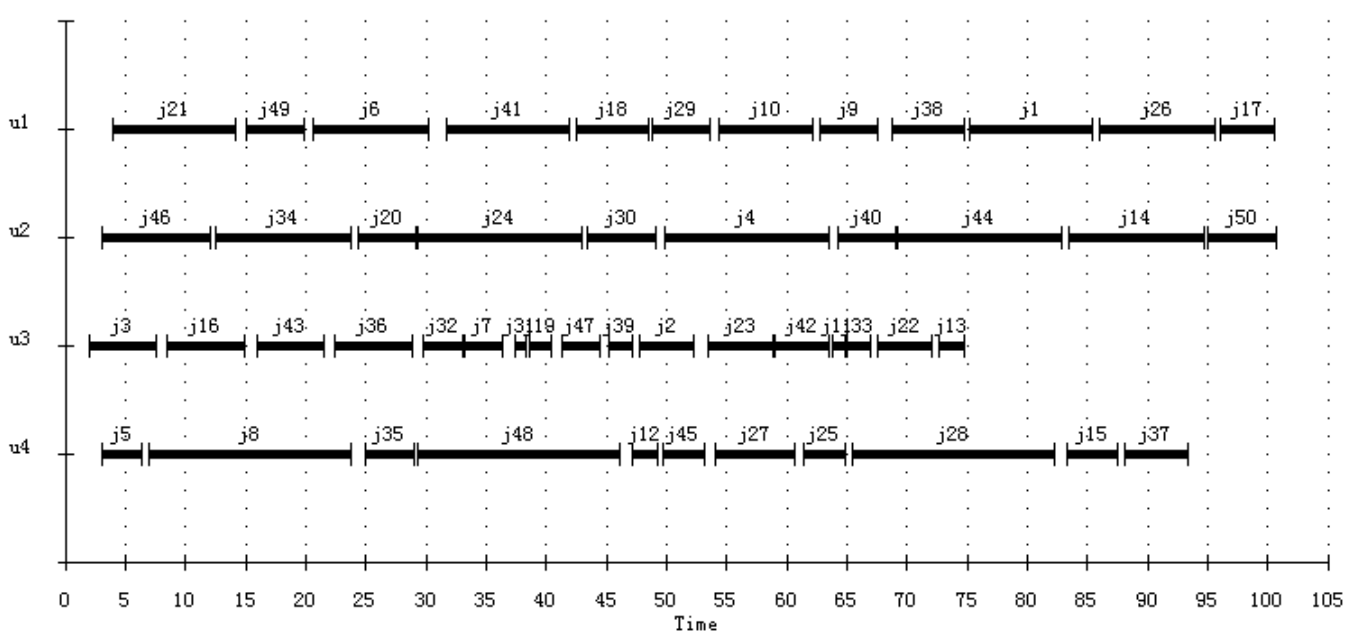

Fig. 13 Gantt chart of a schedule for Example 1b (50 orders over 4 units) by GA (makespan=100.75)

From Fig. 14 and Fig.15, we can obviously see the trend of performances of GA, RS and MILP with the increasing size of the problems. It seems that to solve 50-order problem is already beyond the ability of MILP, but GA is able to get acceptable solution of more large-size problems.

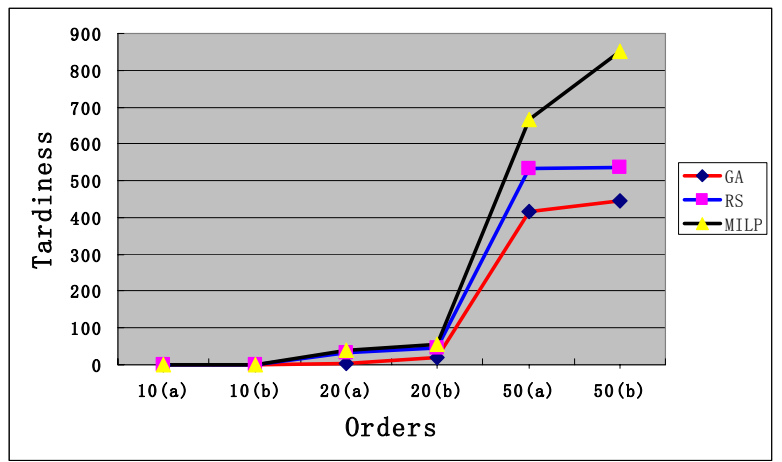

Fig. 14 Contrast of GA, RS and MILP for Example 1 (tardiness) 


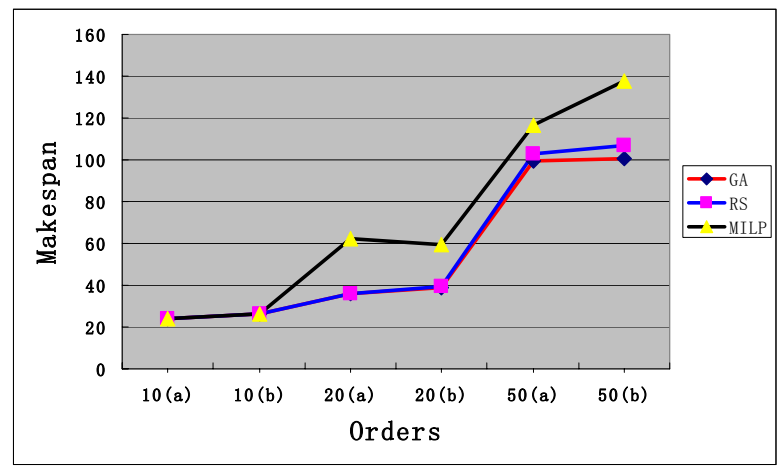

Fig. 15 Contrast of GA, RS and MILP for Example 1 (makspan)

\section{Further works}

\subsection{Improvement to solution technique}

It seems that to solve 50-order problem is already beyond the ability of MILP, but GA is able to get acceptable solution of more large-size problems. As contrast, GA was applied to a very large instance without CP constraints (Example 2), an instance that is hard to be solved by MILP.

With the problem size increasing further, GA will also meet its limited search ability. For 100-order problem, GA (combining with Rule1) was only able to acquire a makespan round about 106.39 (see Fig. 16). However, the best makespan acquired by long-time tabu search (TS) (combining with Rule1) was 99.73, as shown in Fig. 17. The reason for this is that, with the problem size increasing, the solution quality of GA degrades rapidly, that is to say, GA gets into premature convergence, and cannot obtain the near-optimal solution.

Therefore, in order to get much better solutions for super-size problem, measures should be taken to improve the GA, or other solution technique, like tabu search, should be adopted.

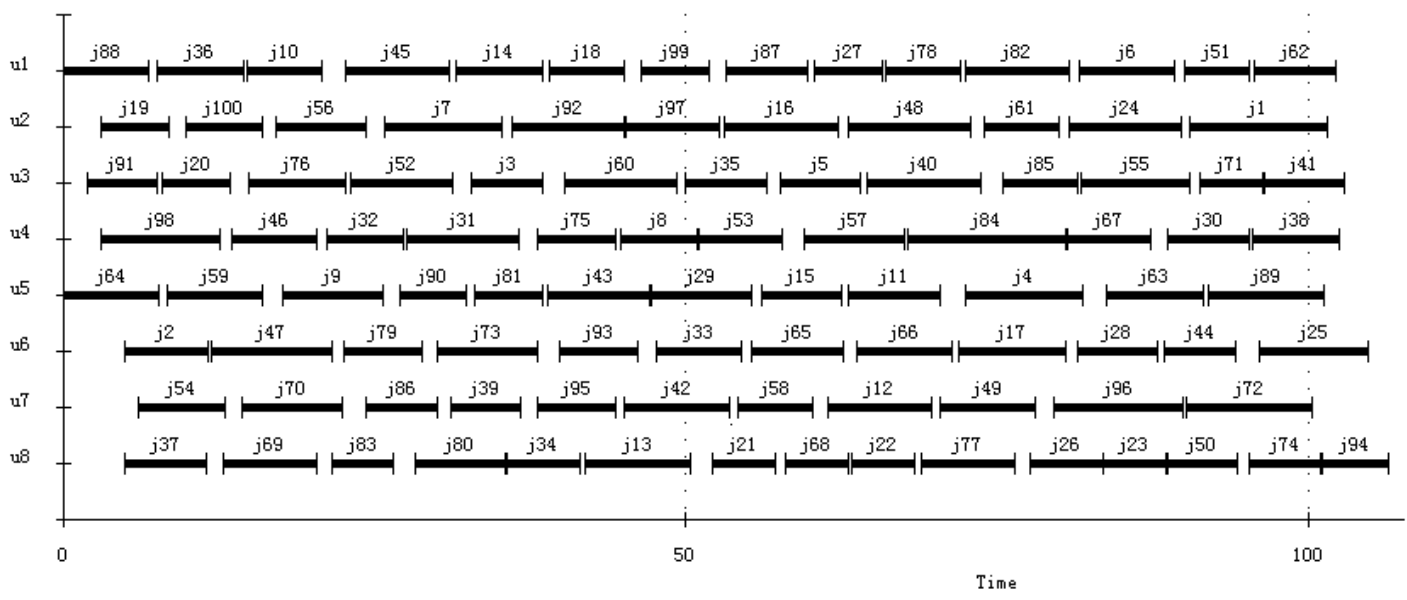

Fig. 16 Gantt chart of a schedule for Example 2 (100 orders over 8 units) by GA (makespan=99.73) 


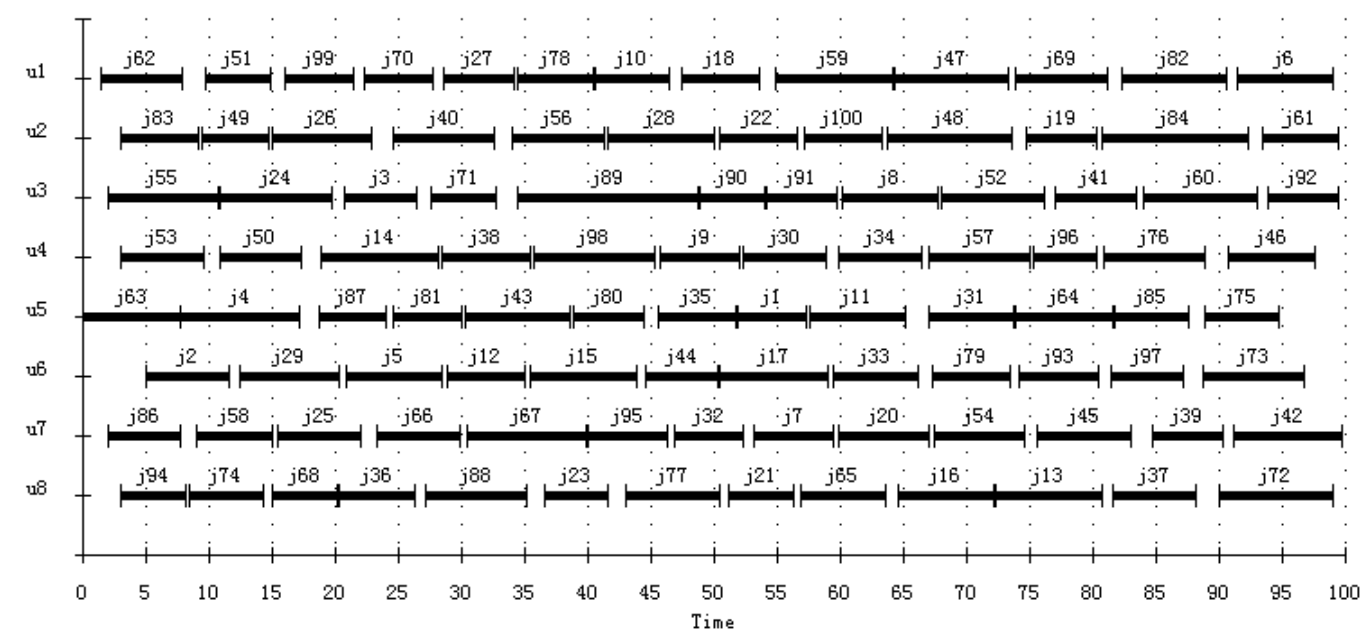

Fig. 3-20 Gantt chart of a schedule for Example2 (100 orders over 8 units) by long-time TS (makespan=99.73)

\subsection{Rule Selection}

In our GA, a suitable rule has been selected to synthesize an order string (a chromosome) into a schedule. Through computation experiments, it is found that, to minimize makespan or total tardiness of SMSP with CP constraints, as Example 1, Rule1 is the best rule in the candidate rule base; but to SMSP without CP constraints, as Example 2, Rule6 is the best rule. Table 9 is the computation results of Example 2 by GA and TS combining with Rule 1 and Rule 6 respectively. These two methods combining with Rule 6 find better solutions than that with Rule 1 .

For different scheduling objectives, like makespan, total tardiness, total earliness and so on, there are different heuristic rules to be employed. The effectiveness of a heuristic rule depends on the scheduling objective and the prevailing shop or plant conditions.

In practice, in order to select a suitable rule from a candidate rule base, considerable simulation experiments have to be conducted. So we wonder, whether rules can be chosen automatically by the algorithm itself, according to different scheduling objective, problem type and the prevailing shop or plant conditions, or not? The answer is positive. We have developed a novel genetic algorithm-based method that evolves scheduling rules while evolving the solutions. Throughout the genetic evolution, a suitable heuristic rule or rule sequence will be automatically selected.

Our subsequent publications will include these further research works in detail.

\section{Conclusions}

MILP is not suitable to solve large-size SMSP. In this paper, MILP has been applied to large-size instances. It is found that: even if the algorithm was allowed to run for a great number of iterations, the solution finally achieved is far from satisfaction.

For small-size instances, random search based on heuristic rule can also acquire optimal solution as MILP do. For large-size instances, random search based on heuristic rule outperforms MILP. Actually, the initial step of GA to generate random solution is a process of random search. 
GA was able to get acceptable solutions of the large-size instances within moderate time. Heuristic rule plays a very important role in RS and GA. If poor heuristic rule is adopted in the algorithm, the solution will also be poor.

Due to the CP constraints in SMSP, it will take GA a plenty of time to generate feasible solutions, increasing the computational time greatly. However, by substituting the forbidden changeovers and processes with a large penalty numerical value, the problem is changed to be without CP constraints. By this penalty method, the search time of GA to large-size high-constrained problem is reduced greatly. Hence, GA is suitable for large-size high-constrained problem.

With the problem size increasing further, GA will more easily get into premature convergence. In order to get much better solutions for super-size problem, measures should be taken to improve the GA, or other solution technique, like tabu search, should be adopted.

\section{Nomenclature}

\section{(a) Indices}

$i, j=$ different customer orders

$u=$ processing units

$t=$ iterations of the algorithm

\section{(b) Sets}

$O=$ a set of orders (with $N$ orders)

$U=$ a set of units (with $M$ units)

\section{(c) Parameters}

$N=$ the number of orders

$M=$ the number of units

$c_{i j}=$ changeover time when order $i$ changeover to order $j$ (not unit dependant)

$o r_{j}=$ order release time: the earliest time at which order $j$ can start its processing

$u r_{u}=$ unit release time: the represents the time at which unit $u$ can get ready

$d_{j}=$ due date: the committed shipping or completion date of order $j$ (the date the order is promised

to the customer)

popsize $=$ the number of random solutions, the number of chromosomes in the initial generation

$x$ size $=$ the number of the chromosomes that are selected to crossover

$m s i z e=$ the number of the chromosomes that are selected to mutate

$C_{r}=$ crossover rate

$M_{r}=$ mutation rate

$C P U, C P U$ time $=$ computation time of the algorithm

$Q_{j}=$ the size of order $j$,

$B_{j u}=$ batch size of order $j$ in the unit $u$

$b_{j u}=$ batch time of order $j$ in the unit $u$

$p_{j u}=$ order process time

\section{(d) Variables and functions}


$C_{j}=$ completion time of order $j$

$T_{j}=$ tardiness of job $j$

$C_{\max }=$ makespan

$T=$ total tardiness

$\pi=$ a permutation $\left(\pi_{1}, \pi_{2}, \ldots, \pi_{N}\right)$ in set $O$

$f(\pi)=$ objective value of $\pi$ 


\section{References}

Cafaro, D. C., \& Cerda, J. (2004). Optimal scheduling of multiproduct pipeline systems using a nondiscrete MILP formulation. Computers \& Chemical Engineering, 28, 2053-2068.

Cerda, J., Henning, P., \& Grossmann, I. E. (1997). A mixed integer linear programming model for short-term scheduling of single-stage multiproduct batch plants with parallel lines. Industrial \& Engineering Chemistry Research, 36, 1695-1707.

Chen, C., Liu, C., Feng, X., \& Shao H. (2002). Optimal short-term scheduling of multiproduct single-stage batch plants with parallel lines. Industrial \& Engineering Chemistry Research, 41, 1249-1260.

Chen, C., Neppalli, R. V., \& Aljaber N. (1996). Genetic algorithms applied to the continuous flow shop problem. Computers \& Industrial Engineering, 30, 919-929.

Das, H., Cummings, P. T., \& Le Van, M. D. (1991). Scheduling of serial multiproduct batch processes via simulated annealing. Computers \& Chemical Engineering, 14, 1351-1362.

Gen, M., \& Cheng, R. (1997). Genetic algorithms and engineering design. Wiley, New York.

Ghaeli, M., Bahri, P. A., Lee, P., \& Gu T. (2005). Petri-net based formulation and algorithm for short-term scheduling of batch plants. Computers \& Chemical Engineering, 29, 249-259.

Goldberg, D. E., \& Deb, K. (1991). A comparative analysis of selection schemes used in genetic algorithms. In: Rawlins, G. (Eds.), Foundations of genetic algorithms, Morgan Kaufmann.

Goldberg, D. E.(1989). Genetic algorithms in search, optimisation and machine learning. Addison-Wesley (Reading, Mass).

Hui, C. W., Gupta, A., \& Meulen H. (2000). A novel MILP formulation for short term scheduling of multistage multi-products batch plants with sequence-dependent constraints . Computers \& Chemical Engineering, 24, 1611-1617.

Hui, C. W., \& Gupta, A.( 2001). A bi-index continuous time MILP model for Short-term scheduling of single-stage multi-product batch plants with parallel line. Industrial \& Engineering Chemistry Research, 40, 5960-5967.

Ierapetritou, M. G., \& Floudas, C. A. (1998a). Effective continuous time formulation for short-term scheduling. 1. Multipurpose batch processes. Industrial \& Engineering Chemistry Research, 37, 4341-4359.

Ierapetritou, M. G., \& Floudas, C. A. (1998b). Effective continuous-time formulation for short-term scheduling: 2. Continuous and semicontinuous processes. Industrial \& Engineering Chemistry Research, 37, 4360-4374.

Jou, C. (2005). A genetic algorithm with sub-indexed partitioning genes and its application to production scheduling of parallel machines. Computers \& Industrial Engineering, 48, 39-54.

Karimi, I. A., \& McDonald, C. M. (1997). Planning and scheduling of parallel semi-continuous process. 2. short-term scheduling. Industrial \& Engineering Chemistry Research, 36, 2701-2714.

Kim, M. S., Jang, J. H., \& Lee, I. B. (1996). Intelligent scheduling and monitoring for multi-product networked batch processes. Computers \& Chemical Engineering, 20, 1149-1154.

Ku, H. M., \& Karimi, I. A. (1991). An Evaluation of Simulated Annealing for Batch Process Scheduling. Industrial \& Engineering Chemistry Research, 30, 163-169.

Lee, D. S., Vassiliadis, V. S., \& Park, J. M. (2002). List-based threshhold-accepting algorithm for zero-wait scheduling of multiproduct batch plants. Industrial \& Engineering Chemistry 
Research, 42, 6579-6588.

Mendez, C. A., \& Cerda J. (2000a). Optimal scheduling of a resource-constrained multiproduct batch plant supplying intermediates to nearby end-product facilities. Computers \& Chemical Engineering, 24, 369-376

Mendez, C. A., Henning, G. P., \& Cerda, J. (2000b). Optimal scheduling of batch plants satisfying multiple product orders with different due-dates. Computers \& Chemical Engineering, 24, 2223-2245.

Mockus, L., \& Reklaitis, G. V. (1996). Continuous time presentation in batch/semicontinuous process scheduling: randomized heuristic approach. Computers \& Chemical Engineering, 20, $1173-1177$.

Pinto, J. M., \& Grossmann, I. E. (1996). A continuous time MILP model for short term scheduling of batch plants with pre-ordering constraints. Computers \& Chemical Engineering, 20, P1197-1202.

Pinto, J. M., \& Grossmann, I. E. (1995). A continuous time mixed integer linear programming model for short term scheduling of multistage batch plants. Industrial \& Engineering Chemistry Research, 34, 3037-3051.

Pinto, J. M., \& Grossmann, I. E. (1998). Assignment and sequencing models for the scheduling of process systems. Annals of Operations Research, 81, 433- 466.

Pinto, J. M., Turkay, A., Bolio, B., \& Grossmann, I. E. (1998). STBS: A continuous-time MILP optimization for short-term scheduling of batch plants. Computers \& Chemical Engineering, 22, 1297-11308.

Poon, P. W., \& Carter, J. N. (1995). Genetic algorithm crossover operators for ordering applications. Computers \& Operations Research, 22, 135-47.

Reklaitis, G. V., \& Mokus L. (1995). Mathematical programming formulation for scheduling of batch operations based on non uniform time discretization. Acta Chimica Slovenica, 42, 81-86.

Wu, D., \& Ierapetritou, M. G. (2004). Cyclic short-term scheduling of multiproduct batch plants using continuous-time representation. Computers \& Chemical Engineering, 28, 2271-2286. 


\section{Appendix Data of Example 1}

Table 1 Order size $\left(Q_{j}\right)$, order due date $\left(d_{j}\right)$ and order release time $\left(\right.$ or $\left._{j}\right)$

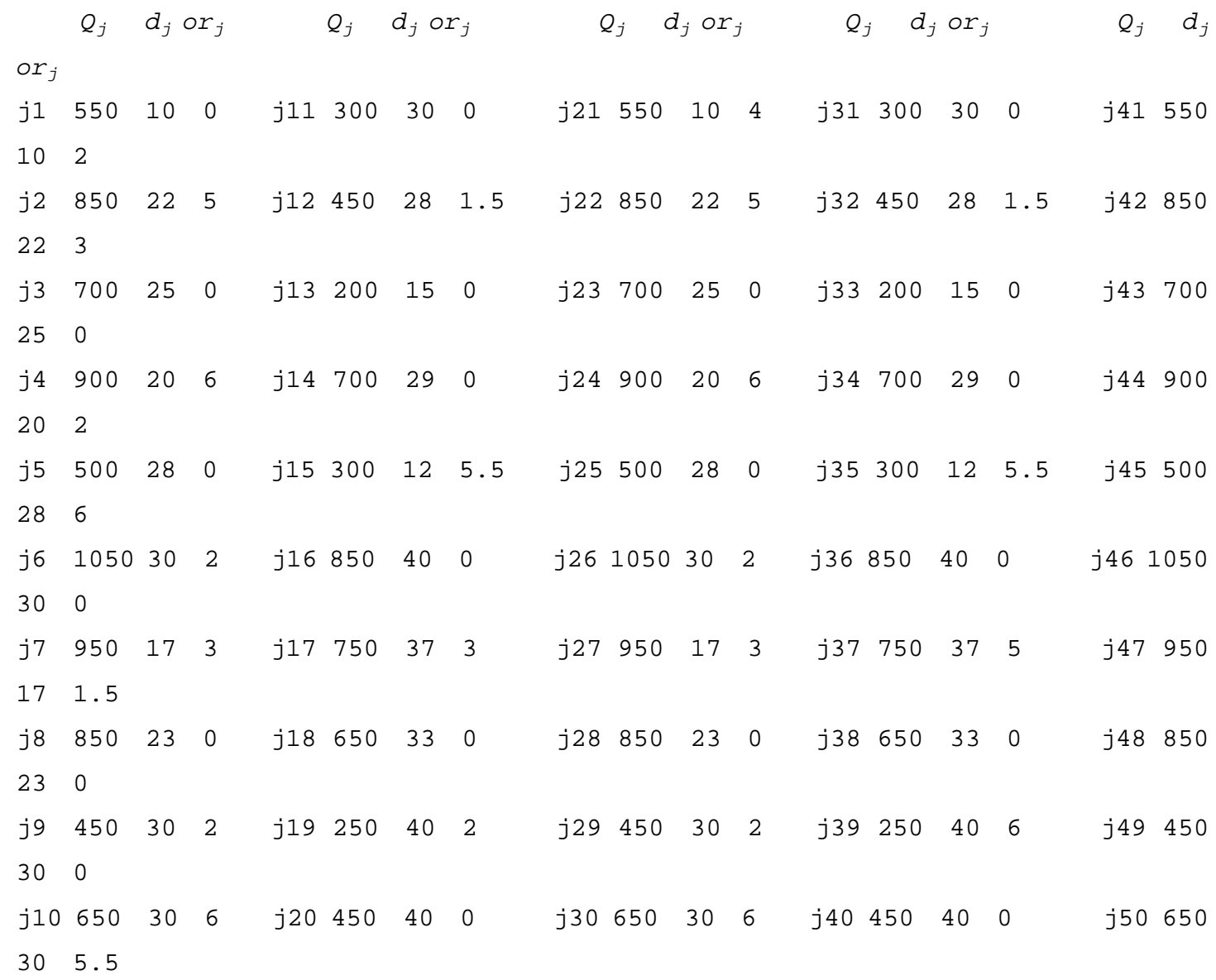

Table 2 Batch size $\left(B_{j u}\right)$ of the orders in the various units

\begin{tabular}{|c|c|c|c|c|c|c|c|c|c|c|c|c|c|c|}
\hline & u1 & $\mathrm{u} 2$ & u3 & $\mathrm{u} 4$ & & u1 & $\mathrm{u} 2$ & u3 & u4 & & u1 & $\mathrm{u} 2$ & u3 & u4 \\
\hline j1 & 100 & & & & j18 & 155 & 160 & & & j35 & & 130 & & 145 \\
\hline j2 & & & 210 & & j19 & & 205 & 170 & & j36 & & & 185 & \\
\hline j3 & 140 & & 170 & & j20 & & 120 & & 145 & j37 & 110 & & & 165 \\
\hline j4 & & 120 & & & j21 & 100 & & & & j38 & 155 & $16 \odot$ & & \\
\hline j5 & & 90 & & 130 & j22 & & & 210 & & j39 & & 205 & 170 & \\
\hline j6 & 280 & 210 & & & j23 & 140 & & 170 & & $j 40$ & & 120 & & 145 \\
\hline j7 & & & 390 & 290 & j24 & & 120 & & & j41 & 100 & & & \\
\hline j8 & & & & 120 & j25 & & $9 \odot$ & & 130 & j42 & & & 210 & \\
\hline j9 & $20 \odot$ & & & & j26 & 280 & 210 & & & $\mathrm{j} 43$ & 140 & & 170 & \\
\hline j10 & 250 & 270 & & & j27 & & & 390 & $29 \odot$ & j44 & & 120 & & \\
\hline j11 & & & 190 & & j28 & & & & 120 & j45 & & 90 & & 130 \\
\hline j12 & & & 140 & 150 & j29 & $20 \odot$ & & & & j46 & 280 & 210 & & \\
\hline j13 & 120 & & 155 & & j30 & 250 & 270 & & & j47 & & & 390 & 290 \\
\hline j14 & & 115 & & & j31 & & & 190 & & $\mathrm{j} 48$ & & & & 120 \\
\hline j15 & & 130 & & 145 & j32 & & & 140 & 150 & j49 & $20 \odot$ & & & \\
\hline j16 & & & 185 & & j33 & 120 & & 155 & & j50 & 250 & 270 & & \\
\hline j17 & 110 & & & 165 & j34 & & 115 & & & & & & & \\
\hline
\end{tabular}


Table 3 Batch time $\left(b_{j u}\right)$ and unit release time $\left(u r_{u}\right)$

\begin{tabular}{|c|c|c|c|c|c|c|c|c|c|c|c|c|c|c|}
\hline & u1 & u2 & u3 & u4 & & u1 & $\mathrm{u} 2$ & u3 & u4 & & u1 & u2 & u3 & u4 \\
\hline j1 & 1.7 & & & & j18 & 1.2 & 1.45 & & & j35 & & 1.48 & & 1.39 \\
\hline j2 & & & $\odot .9$ & & j19 & & 1.85 & $\odot .95$ & & j36 & & & 1.3 & \\
\hline j3 & 1.25 & & 1.1 & & j20 & & 1.2 & & 1.3 & j37 & 0.65 & & & 1.05 \\
\hline j4 & & 1.7 & & & j21 & 1.7 & & & & j38 & $1.2=$ & 1.45 & & \\
\hline 5 & & 1.4 & & $\odot .85$ & j22 & & & $\odot .9$ & & j39 & & 1.85 & 0.95 & \\
\hline j6 & 2.4 & 1.8 & & & j23 & 1.25 & & 1.1 & & j40 & & 1.2 & & 1.3 \\
\hline j7 & & & 1.05 & 1.65 & j24 & & 1.7 & & & j41 & 1.7 & & & \\
\hline j8 & & & & 2.1 & j25 & & 1.4 & & $\odot .85$ & j42 & & & 0.9 & \\
\hline j9 & 1.6 & & & & j26 & 2.4 & 1.8 & & & $j 43$ & 1.25 & & 1.1 & \\
\hline j10 & 2.6 & 1.9 & & & j27 & & & 1.05 & 1.65 & j44 & & 1.7 & & \\
\hline j11 & & & $\odot .5$ & & j28 & & & & 2.1 & j45 & & 1.4 & & ๑. 85 \\
\hline j12 & & & $\odot .85$ & 0.7 & j29 & 1.6 & & & & j46 & 2.4 & 1.8 & & \\
\hline j13 & 1.75 & & 1.0 & & j30 & 2.6 & 1.9 & & & j47 & & & 1.05 & 1.65 \\
\hline j14 & & 1.6 & & & j31 & & & $\odot .5$ & & j48 & & & & 2.1 \\
\hline j15 & & 1.48 & & 1.39 & j32 & & & 0.85 & 0.7 & j49 & 1.6 & & & \\
\hline j16 & & & 1.3 & & j33 & 1.75 & & 1.0 & & j50 & 2.6 & 1.9 & & \\
\hline j17 & 0.65 & & & 1.05 & j34 & & 1.6 & & & $u r_{u}$ & 0 & 3 & 2 & 3 \\
\hline
\end{tabular}

Table 4 Changeover time $\left(c_{i j}\right)$ of order pairs

$\begin{array}{llllllllllllllllll} & j 1 & j 2 & j 3 & j 4 & j 5 & j 6 & j 7 & j 8 & j 9 & j 10 & j 11 & j 12 & j 13 & j 14 & j 15 & j 16 & j 17 \\ i 1 & - & - & - & - & - & .65 & - & - & .85 & .4 & - & - & .35 & - & - & - & - \\ i 2 & - & - & 1.1 & - & - & - & - & - & - & - & .25 & - & .7 & - & - & .25 & - \\ i 3 & 1.0 & .15 & - & - & - & - & .3 & - & 1.6 & .2 & .5 & .75 & - & - & - & .9 & .6 \\ i 4 & - & - & - & - & .05 & - & - & - & - & .5 & - & - & - & .7 & .45 & - & - \\ i 5 & - & - & - & .3 & - & .7 & .9 & .6 & - & - & - & .9 & - & .8 & - & - & - \\ i 6 & 1.4 & - & .3 & .7 & - & - & - & - & 1.2 & - & - & - & 1.2 & .55 & .2 & - & .35 \\ i 7 & - & 1.8 & - & - & .85 & - & - & .45 & - & - & 1.0 & 1.1 & - & - & - & .8 & .5 \\ i 8 & - & - & - & - & - & - & 1.65 & - & - & - & - & 1.05 & - & - & 1.10 & - & - \\ i 9 & 2.1 & - & 1.25 & - & - & .8 & - & - & - & .65 & - & - & .85 & - & - & - & .15 \\ i 10 & 1.5 & - & .6 & .75 & .5 & - & - & - & .7 & - & - & - & 1.15 & 1.3 & .95 & - & .4 \\ i 11 & - & .95 & - & - & - & - & - & - & - & - & - & .15 & .15 & - & - & .35 & - \\ i 12 & - & - & .8 & - & .4 & - & .1 & .2 & - & - & .6 & - & - & - & 1.3 & 1.0 & .8 \\ i 13 & .3 & .55 & 1.3 & - & - & 1.3 & 1.55 & - & .25 & 1.15 & 1.4 & .4 & - & - & - & .5 & .25 \\ i 14 & - & - & - & 1.45 & .8 & .5 & - & - & - & .35 & - & - & - & - & .75 & - & - \\ i 15 & - & - & - & .2 & - & .4 & 1.2 & .3 & - & .8 & - & .3 & - & 1.05 & - & - & .6 \\ i 16 & - & .25 & 1.05 & - & - & - & - & - & - & - & - & .85 & .2 & - & - & - & - \\ i 17 & - & - & .8 & - & .3 & .9 & 1.1 & .5 & - & .75 & - & - & .45 & - & .2 & - & - \\ i 18 & .4 & - & - & .5 & .45 & - & - & - & .35 & .6 & - & - & - & .65 & .55 & - & .3 \\ i 19 & - & .7 & - & - & .65 & .85 & .8 & - & - & - & .7 & .9 & - & .5 & 1.05 & .75 & - \\ i 20 & - & - & - & .15 & - & - & .55 & .45 & - & .4 & - & - & - & .4 & - & - & - \\ i 21 & - & - & - & - & - & .65 & - & - & .85 & .4 & - & - & .35 & - & - & - & -\end{array}$




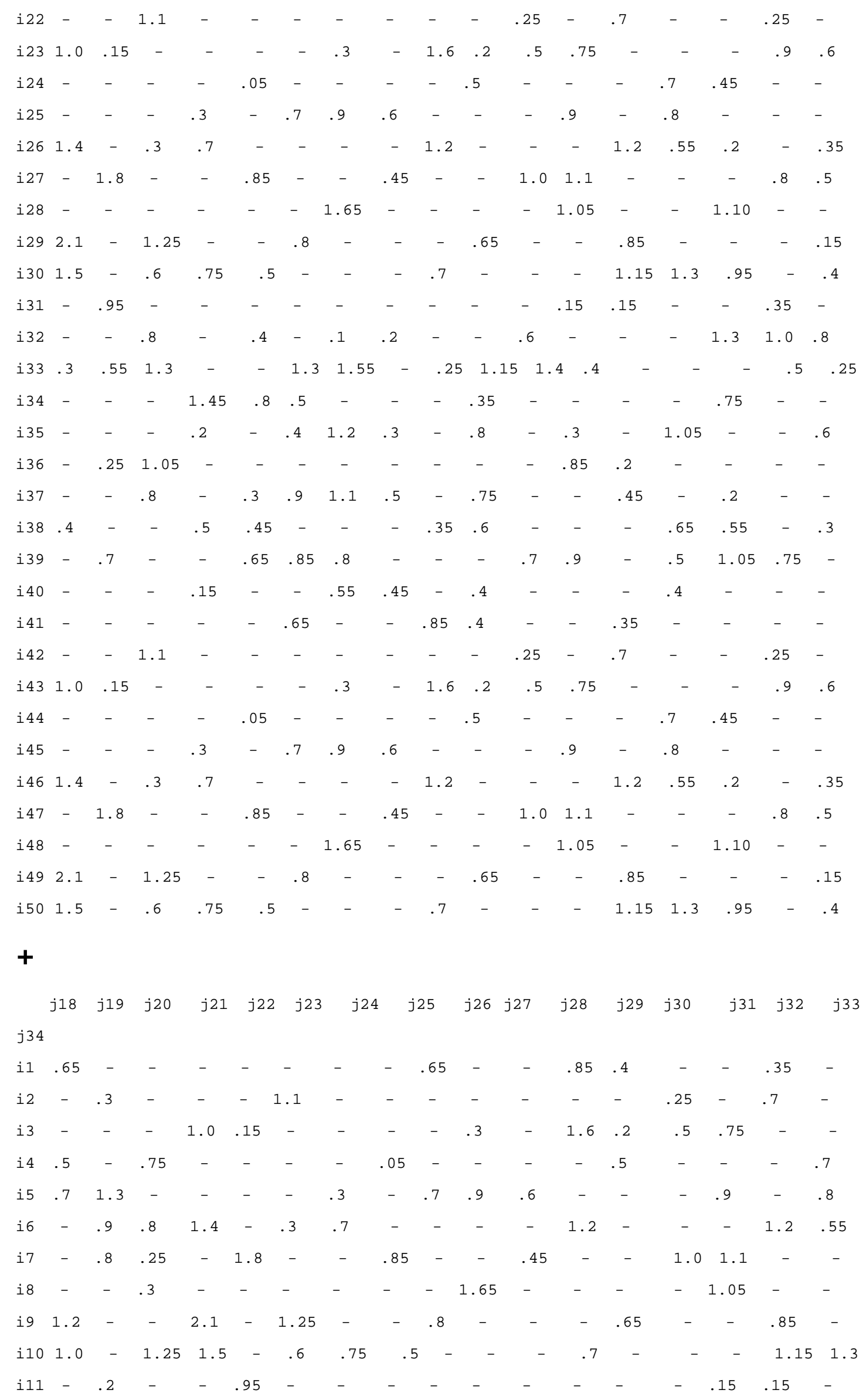




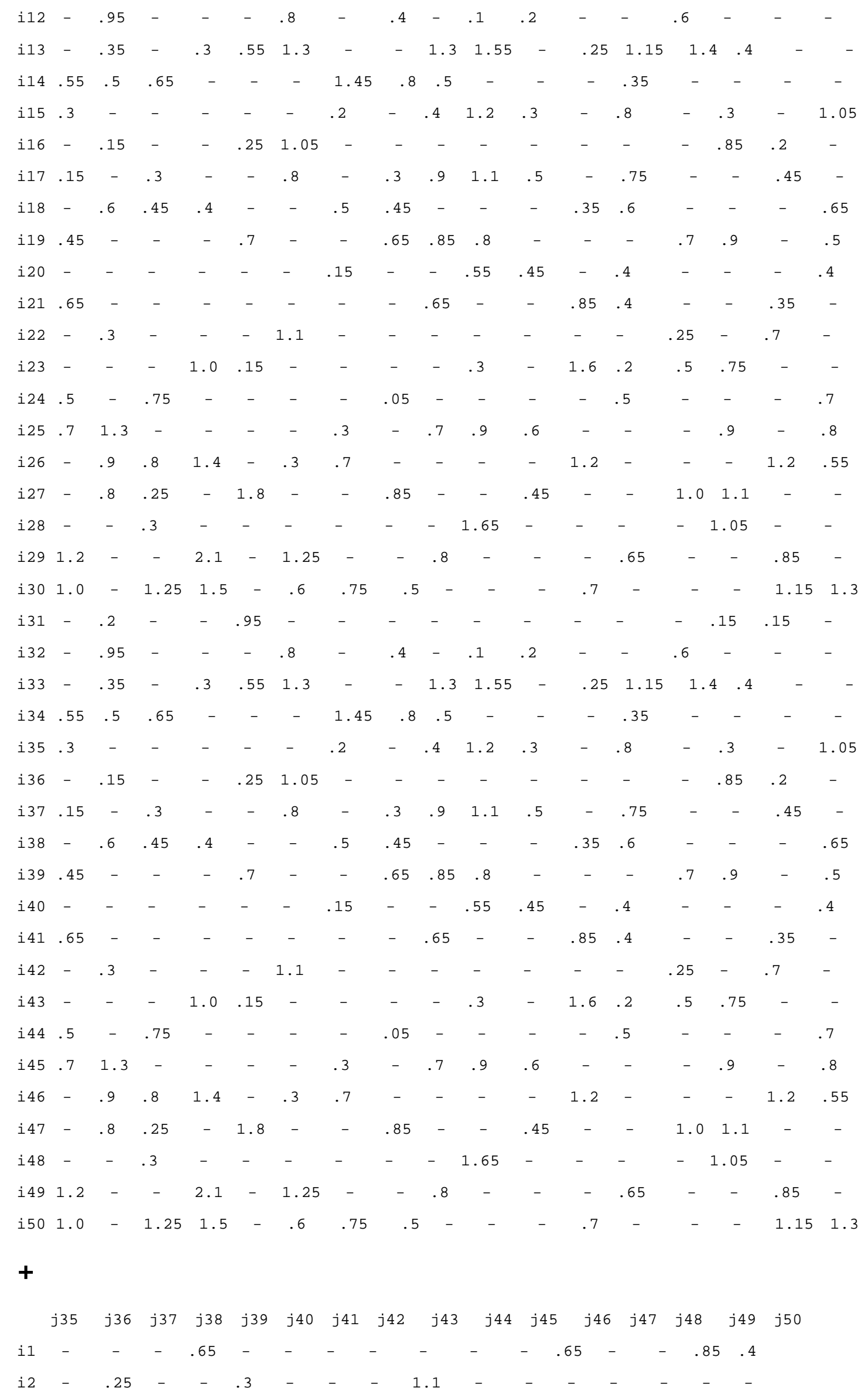




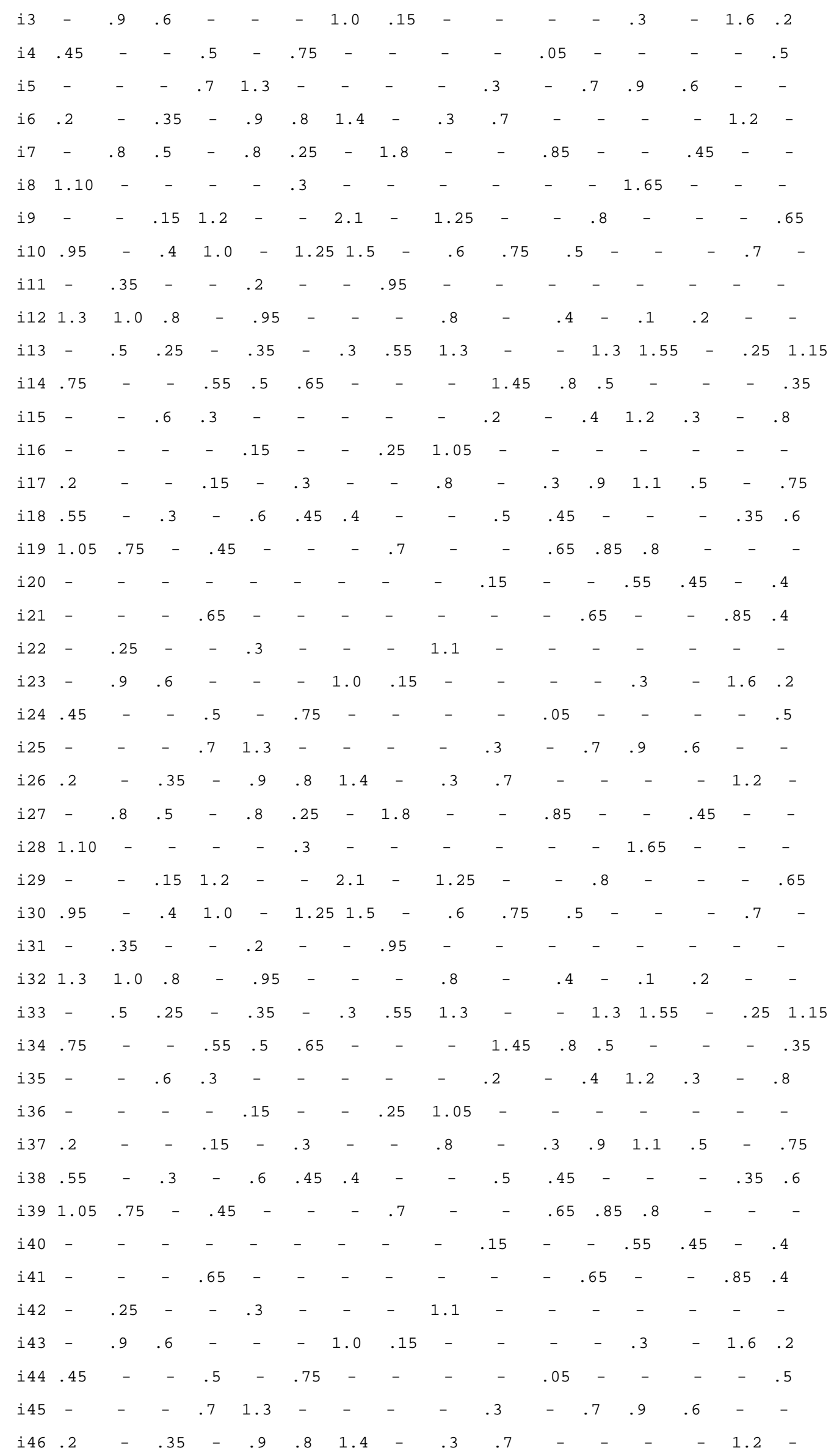




$\begin{array}{llllllllllllllllll}i 47 & - & .8 & .5 & - & .8 & .25 & - & 1.8 & - & - & .85 & - & - & .45 & - & - \\ i 48 & 1.10 & - & - & - & - & .3 & - & - & - & - & - & - & 1.65 & - & - & - \\ i 49- & - & .15 & 1.2 & - & - & 2.1 & - & 1.25 & - & - & .8 & - & - & - & .65 \\ i 50 & .95 & - & .4 & 1.0 & - & 1.25 & 1.5 & - & .6 & .75 & .5 & - & - & - & .7 & -\end{array}$

Table 5 Results of Example 1 by MILP

(Case a: Null release time, Case b: Finite release time)

\begin{tabular}{|c|c|c|c|c|}
\hline Orders & $\begin{array}{l}\text { Objective } \\
\text { function }\end{array}$ & Iterations & $\begin{array}{l}\text { CPU time } \\
\text { (seconds) }\end{array}$ & $\begin{array}{c}\text { Objective } \\
\text { value }\end{array}$ \\
\hline \multirow{2}{*}{$10(a)$} & Tardiness & 417 & 0.09 & 0.2 \\
\hline & Makespan & 9542 & 1.55 & 23.90 \\
\hline \multirow{2}{*}{$10(\mathrm{~b})$} & Tardiness & 2471 & 0.39 & 1.00 \\
\hline & Makespan & 3153 & 0.51 & 26.25 \\
\hline \multirow{2}{*}{$20(a)$} & Tardiness & 100000 & 36.76 & 40.42 \\
\hline & Makespan & 100000 & 36.96 & 62.44 \\
\hline \multirow{2}{*}{ 20(b) } & Tardiness & 100000 & 44.25 & 55.29 \\
\hline & Makespan & 100000 & 34.28 & 59.24 \\
\hline \multirow{4}{*}{$50(a)$} & \multirow{2}{*}{ Tardiness } & 100000 & 180.98 & 664.78 \\
\hline & & 1000000 & 1701.43 & 659.48 \\
\hline & \multirow{2}{*}{ Makespan } & 100000 & 166.75 & 116.40 \\
\hline & & 1000000 & 1632.51 & 116.40 \\
\hline \multirow{4}{*}{$50(b)$} & \multirow{2}{*}{ Tardiness } & 100000 & 169.35 & 852.31 \\
\hline & & 1000000 & 1808.66 & 659.20 \\
\hline & \multirow{2}{*}{ Makespan } & 100000 & 158.62 & 137.65 \\
\hline & & 1000000 & 1414.53 & 137.65 \\
\hline
\end{tabular}

The computation tests were run on computer with Intel $(R)$

Pentium (R) M 1500MHz CPU and 256M memory.

Table 6 Results of Example 1 by random search and MILP

\begin{tabular}{ccccccc}
\hline \multirow{2}{*}{ Orders } & Objective & \multicolumn{3}{c}{ Random Search } & \multicolumn{2}{c}{ MILP } \\
\cline { 3 - 7 } & popsize & $\begin{array}{c}\text { CPU time } \\
\text { (seconds) }\end{array}$ & $\begin{array}{c}\text { Objective } \\
\text { value }\end{array}$ & $\begin{array}{c}\text { CPU time } \\
\text { (seconds) }\end{array}$ & $\begin{array}{c}\text { Objective } \\
\text { value }\end{array}$ \\
\hline \multirow{2}{*}{$10(\mathrm{a})$} & Tardiness & 200 & $<\mathbf{1}$ & $\mathbf{0 . 2 0}$ & $\mathbf{0 . 0 9}$ & $\mathbf{0 . 2 0}$ \\
\cline { 2 - 7 } & Makespan & 200 & $<\mathbf{1}$ & $\mathbf{2 3 . 9}$ & $\mathbf{1 . 5 5}$ & $\mathbf{2 3 . 9 0}$ \\
\hline \multirow{2}{*}{$10(\mathrm{~b})$} & Tardiness & 200 & $<\mathbf{1}$ & $\mathbf{1 . 0 0}$ & $\mathbf{0 . 3 9}$ & $\mathbf{1 . 0 0}$ \\
\cline { 2 - 7 } & Makespan & 200 & $<\mathbf{1}$ & $\mathbf{2 6 . 2 5}$ & $\mathbf{0 . 5 1}$ & $\mathbf{2 6 . 2 5}$ \\
\hline
\end{tabular}




\begin{tabular}{ccccccc}
\hline \multirow{2}{*}{ 20(a) } & Tardiness & 200 & $<\mathbf{1}$ & $\mathbf{3 0 . 8 8}$ & $\mathbf{3 6 . 7 6}$ & $\mathbf{4 0 . 4 2}$ \\
\cline { 2 - 7 } & Makespan & 200 & $<\mathbf{1}$ & $\mathbf{3 5 . 8 0}$ & $\mathbf{3 6 . 9 6}$ & $\mathbf{6 2 . 4 4}$ \\
\hline \multirow{2}{*}{ 20(b) } & Tardiness & 200 & $\mathbf{1 . 0 0}$ & $\mathbf{4 6 . 2 7}$ & $\mathbf{4 4 . 2 5}$ & $\mathbf{5 5 . 2 9}$ \\
\cline { 2 - 7 } & Makespan & 200 & $<\mathbf{1}$ & $\mathbf{3 9 . 3 9}$ & $\mathbf{3 4 . 2 8}$ & $\mathbf{5 9 . 2 4}$ \\
\hline \multirow{2}{*}{$50(\mathrm{a})$} & Tardiness & 300 & $\mathbf{2 1 2 . 0 0}$ & $\mathbf{5 3 2 . 5 5}$ & $\mathbf{1 8 0 . 9 8}$ & $\mathbf{6 6 4 . 7 8}$ \\
\cline { 2 - 7 } & Makespan & 300 & $\mathbf{1 5 3 . 0 0}$ & $\mathbf{1 0 2 . 7 9}$ & $\mathbf{1 6 6 . 7 5}$ & $\mathbf{1 1 6 . 4 0}$ \\
\hline \multirow{2}{*}{$50(\mathrm{~b})$} & Tardiness & 300 & $\mathbf{1 7 8 . 0 0}$ & $\mathbf{5 3 7 . 2 6}$ & $\mathbf{1 6 9 . 3 5}$ & $\mathbf{8 5 2 . 3 1}$ \\
\cline { 2 - 7 } & Makespan & 300 & $\mathbf{1 0 7 . 0 0}$ & $\mathbf{1 0 7 . 1 4}$ & $\mathbf{1 5 8 . 6 2}$ & $\mathbf{1 3 7 . 6 5}$ \\
\hline
\end{tabular}

The algorithm was implemented in Clanguage and the computation tests were run on computer with Intel $(R)$ Pentium $(R)$ M 1500MHz CPU and 256M memory.

Table 7 Results of Example 1 by GA, RS and MILP

(Case a: Null release time, Case b: Finite release time)

\begin{tabular}{|c|c|c|c|c|c|c|c|c|}
\hline \multirow[b]{2}{*}{ Orders } & \multirow[b]{2}{*}{ Objective } & \multicolumn{3}{|c|}{ Genetic Algorithm } & \multicolumn{4}{|c|}{ popsize $=200, \quad x$ size $=160$, } \\
\hline & & Iter. & $\begin{array}{l}\text { CPU time } \\
\text { (seconds) }\end{array}$ & $\begin{array}{l}\text { Obj. } \\
\text { value }\end{array}$ & $\begin{array}{l}\text { CPU time } \\
\text { (seconds) }\end{array}$ & $\begin{array}{l}\text { Obj. } \\
\text { value }\end{array}$ & $\begin{array}{l}\text { CPU time } \\
\text { (seconds) }\end{array}$ & $\begin{array}{l}\text { Obj. } \\
\text { value }\end{array}$ \\
\hline \multirow{2}{*}{ 10(a) } & Tardiness & 4 & $<1$ & 0.2 & $<1$ & 0.20 & 0.09 & 0.20 \\
\hline & Makespan & 6 & $<1$ & 23.90 & $<1$ & 23.9 & 1.55 & 23.90 \\
\hline \multirow{2}{*}{ 10(b) } & Tardiness & 5 & $<1$ & 1.00 & $<1$ & 1.00 & 0.39 & 1.00 \\
\hline & Makespan & 5 & $<1$ & 26.25 & $<1$ & 26.25 & 0.51 & 26.25 \\
\hline \multirow{2}{*}{ 20(a) } & Tardiness & 36 & 1 & 4.12 & $<1$ & 30.88 & 36.76 & 40.42 \\
\hline & Makespan & 23 & 1 & 35.80 & $<1$ & 35.80 & 36.96 & 62.44 \\
\hline \multirow{2}{*}{ 20(b) } & Tardiness & 37 & 1 & 20.83 & 1.00 & 46.27 & 44.25 & 55.29 \\
\hline & Makespan & 31 & 1 & 39.05 & $<1$ & 39.39 & 34.28 & 59.24 \\
\hline \multirow{2}{*}{ 50(a) } & Tardiness & 70 & 3501 & 417.26 & 212.00 & 532.55 & 180.98 & 664.78 \\
\hline & Makespan & 50 & 4033 & 99.19 & 153.00 & 102.79 & 166.75 & 116.40 \\
\hline \multirow{2}{*}{$50(\mathrm{~b})$} & Tardiness & 57 & 2287 & 444.54 & 178.00 & 537.26 & 169.35 & 852.31 \\
\hline & Makespan & 55 & 2935 & 100.75 & 107.00 & 107.14 & 158.62 & 137.65 \\
\hline
\end{tabular}

The algorithm was implemented in $C$ language and the computation tests were run on computer with Intel (R) Pentium (R) M 1500MHz CPU and 256M memory.

Table 8 Results of Example 1 with penalty value by GA

\begin{tabular}{ccccc}
\hline Orders & Objective & Iter. & $\begin{array}{c}\text { CPU time } \\
\text { (seconds) }\end{array}$ & $\begin{array}{c}\text { Obj. } \\
\text { value }\end{array}$ \\
\hline \multirow{2}{*}{$50(\mathrm{a})$} & Tardiness & 50 & 2.0 & 389.15 \\
\cline { 2 - 5 } & Makespan & 67 & 2.0 & $\mathbf{9 8 . 9 9}$ \\
\hline \multirow{2}{*}{$50(\mathrm{~b})$} & Tardiness & 73 & 2.0 & 402.97 \\
\cline { 2 - 5 } & Makespan & 50 & 2.0 & $\mathbf{1 0 1 . 1 0}$ \\
\hline
\end{tabular}

Table 9 Results of Example 2 by GA and TS (Combining with Rule 1 and Rule 6 respectively) 


\begin{tabular}{lcccc}
\hline Method & Objective & Iter. & $\begin{array}{c}\text { CPU time } \\
\text { (seconds) }\end{array}$ & $\begin{array}{c}\text { Obj. } \\
\text { value }\end{array}$ \\
\hline GA (with Rule 1) & makespan & 74 & 10 & 106.39 \\
\hline TS (with Rule 1) & makespan & 10000 & 455 & 99.73 \\
\hline GA (with Rule 6) & makespan & 42 & 2 & 97.61 \\
\hline TS (with Rule 6) & makespan & 10000 & 316 & 94.74 \\
\hline $\begin{array}{l}\text { The algorithm was implemented in C language and the computation } \\
\text { tests were run on computer with Intel (R) Pentium (R) M 1500MHz } \\
\text { CPU and 256M memory. }\end{array}$
\end{tabular}


Heuristic Rule-Based Genetic Algorithm for Large-Size High-Constrained Single-Stage

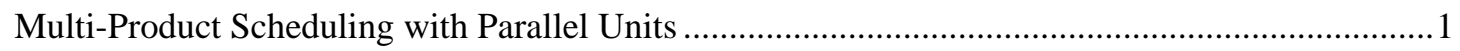

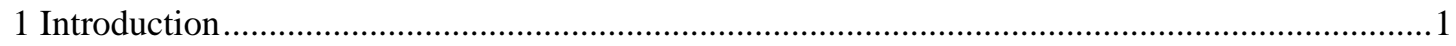

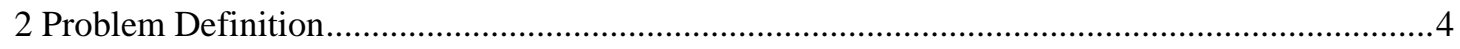

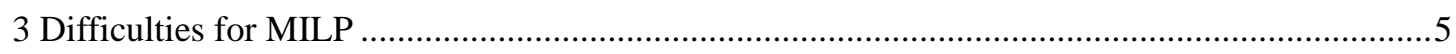

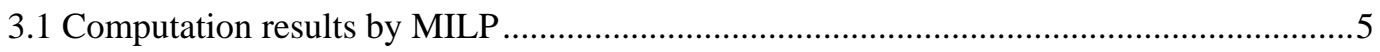

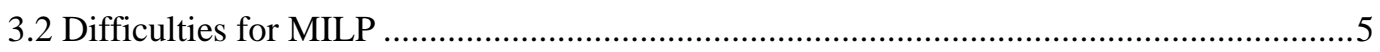

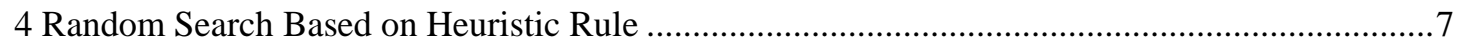

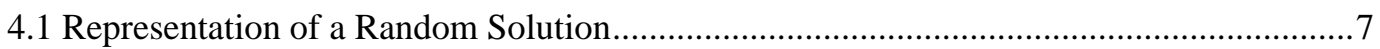

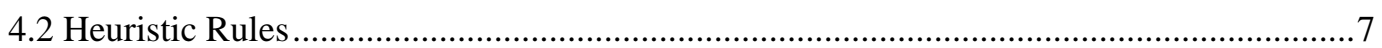

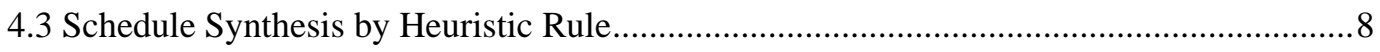

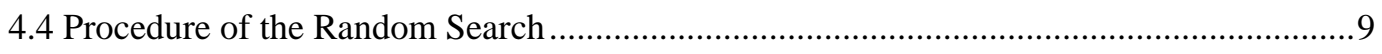

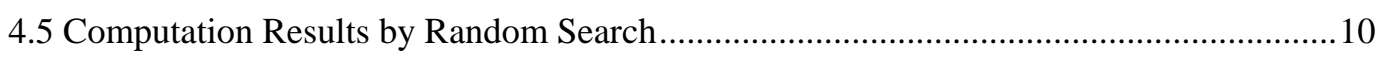

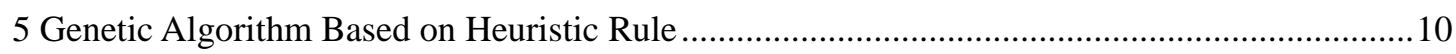

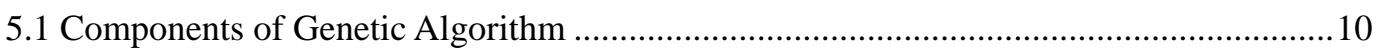

5.1.1 Representation of solutions ................................................................................ 10

5.1.2 Generation ................................................................................................... 11

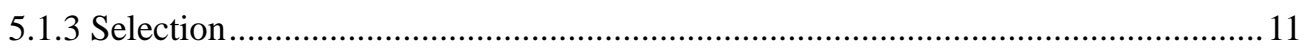

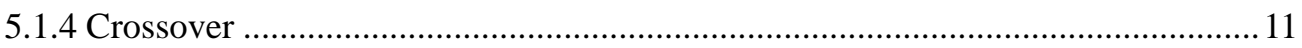

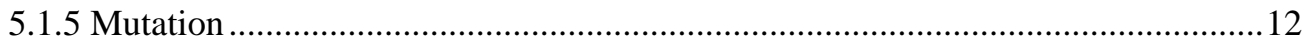

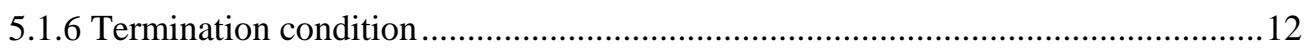

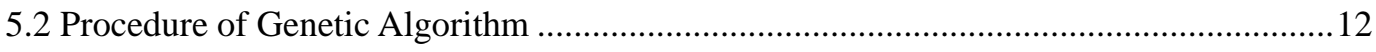

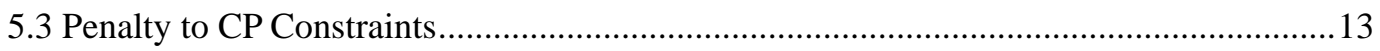

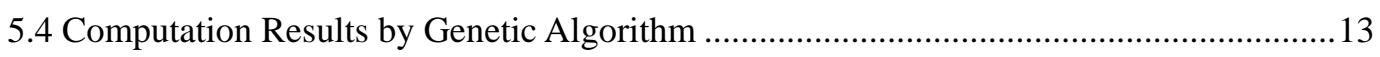

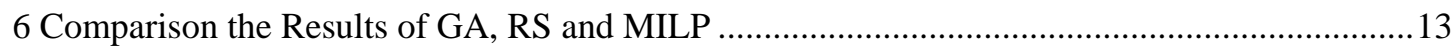

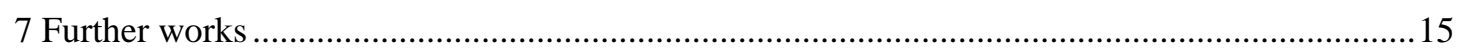

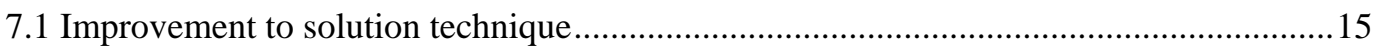

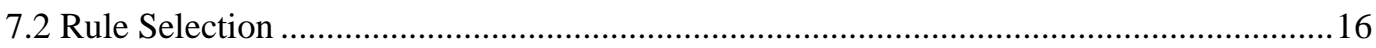

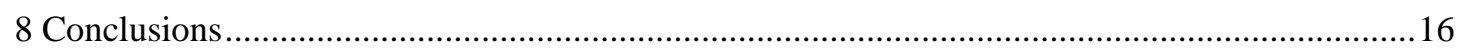

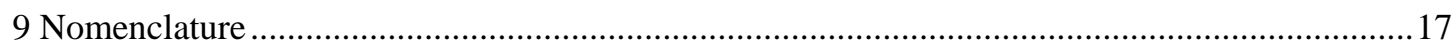

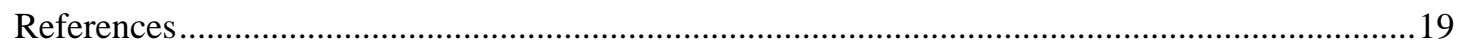

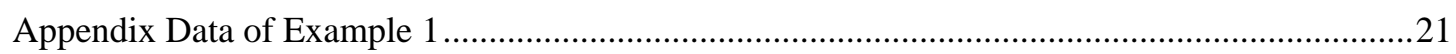

